\title{
LE TERESIANE IN ITALIA: ISTITUZIONI, VITE VISSUTE, AUTORAPPRESENTAZIONI
}

\author{
POR \\ Sara CabibBo \\ Università Roma Tre \\ $\mathrm{Y}$ \\ ElisabetTa MarchetTI \\ Università di Bologna*
}

\section{RESUMEN}

Este artículo se propone reconstruir la rápida difusión que tuvo el Carmelo descalzo en Italia desde los años 80 del s. XVI y durante todo el s. XVII. Mediante la documentación de archivo y los textos impresos durante ese periodo de tiempo, el análisis se detiene en las peculiaridades de las fundaciones italianas y en el debate creado dentro de la Orden sobre los monasterios femeninos y sus Constituciones. A través del análisis de dos monasterios, ambos fundados en la capital siciliana del virreinato español, este estudio se propone además trazar las dinámicas sociales y religiosas que dieron vida a las dos fundaciones palermitanas y, en un sentido más amplio, diseñar el modelo de monja teresiana.

PalabRas ClaVE: Fundaciones carmelitas, monasterios femeninos, constituciones, Teresianas en Palermo (Italia, siglos XVI-XVII).

\section{TERESIAN NUNS IN ITALY: INSTITUTIONS, LIFE STORIES, SELF-REPRESENTATIONS}

\section{ABSTRACT}

This paper analyses the rapid spreading of the Discalced Carmelites Order in Italy from about 1580 to the end of the seventeenth century. Drawing from

\footnotetext{
*Elisabetta Marchetti è autrice dei paragrafi 1-3; Sara Cabibbo dei paragrafi 4-6.
} 
archivistic sources and printed books of the period, the essay focuses on the specificity of the Italian foundations, retracing the debate that took place all along within the Order, about the Carmelite nunneries and their Constitutions. On the basis of the case studies of two nunneries established in the Sicilian capital of the Spanish viceroyalty, the paper outlines the social and religious dynamics which generated the two Sicilian institutions and, more widely, the model of Theresian nun.

KEY WORDS: Discalced Carmelites' Foundations, Nunneries, the Female Carmel in Palerme (Italy, $\mathrm{XVI}^{\text {th }}-\mathrm{XVII}^{\text {th }}$ ).

$\begin{array}{ll}\text { Recibido/Received } & 30-10-2014 \\ \text { Aceptado/Accepted } & 15-12-2014\end{array}$

PREMESSA: DALL'ANTICO ORDINE CARMELITANO ALLE FONDAZIONI DELLE SCALZE

Teresa di Gesù, monaca avilese responsabile del rinnovamento in pieno XVI secolo dell'antica famiglia religiosa del Carmelo, trasmise ai rami maschile e femminile del suo Ordine un marcato anelito missionario grazie al quale Scalzi e Scalze continuarono fin dai primi anni dopo la sua morte (1582) la erezione di nuovi conventi e monasteri dentro e fuori la Spagna, culla della neonata riforma. Studiare il radicarsi della novità teresiana in Italia, tramite concrete realtà fondazionali femminili arricchite da esperienze di vita alla base di originali scritti autobiografici, significa innanzi tutto ricordare che per l'antico Ordine del Carmelo la prima espansione sul territorio italiano comportò una evoluzione nella propria struttura ed organizzazione.

A partire dal 1562 - anno della fondazione ad Avila di San Giuseppe prima casa di Scalze - il rapido incremento del ramo femminile e di quello maschile, fomentò contrasti all'interno del primitivo Ordine carmelitano e opposizioni esterne che indussero il Papato a stabilire nel 1581 l'erezione della Provincia dei Carmelitani scalzi. Tuttavia, persistendo una difficoltà di rapporto e convivenza tra Riforma scalza e Ordine del Carmelo, si giunse ad una totale e definitiva separazione dei due rami - Calzati e Scalzi - sancita il 20 dicembre 1593 dal breve pontificio di Clemente VIII.

Nel frattempo continuava a crescere il numero degli insediamenti dei Teresiani le cui fondazioni si sparsero su gran parte del suolo spagnolo, raggiungendo sin dal 1583-84, sulla scia dello zelo missionario di Teresa, la Guinea, il Congo, il Messico e l'Italia. Se i viaggi e gli insediamenti nei primi tre paesi ottennero, generalmente, l'approvazione dei superiori, l'espansione in Italia suscitò l'opposizione sia di una parte dell'Ordine, poco favorevole al radicamento in terre non spagnole, sia - e in maniera decisa - da parte della Corona. La 
Monarchia infatti appoggiò e sostenne decisamente fin dall'inizio la novità teresiana, considerandola però prerogativa nazionale ed importante vetrina di quella riforma degli ordini religiosi promossa da Filippo II, ${ }^{1}$ responsabile del consolidarsi di una politica volta a riaffermare l'identità nazionale anche mediante la difesa e il rafforzarsi di precise realtà religiose ${ }^{2}$

Dopo elaborate trattative, papa Aldobrandini con il breve In apostolicae dignitatis culmine del 13 novembre 1600 decretava la definitiva separazione ed indipendenza della Congregazione scalza di Italia, detta di Sant'Elia, da quella spagnola denominata di San Giuseppe: una divisione, richiesta esplicitamente dalla monarchia spagnola e approvata dall' Ordine, che confermava anche le linee guida del papato di Clemente VIII, impegnato in una riforma ecclesiale giudicata non più procrastinabile e deciso a sottrarsi all'incombente influenza iberica.

In tale ottica l'appoggio romano all'iniziativa teresiana, specie in ordine all'assestamento sul suolo italiano delle fondazioni scalze, assume un posto significativo nei piani e nei desideri della curia. La divisione delle due Congregazioni prevedeva che si affidassero alla parte spagnola tutti i territori soggetti alla Corona, sia nella penisola iberica come nei suoi domini, mentre alla Congregazione d'Italia erano assegnate, quali zone di missione e diffusione, tutti i paesi del mondo -ubique terrarum - eccetto i territori d'influenza spagnola e portoghese. Nella neonata Congregazione italiana confluirono molti dei primi Scalzi spagnoli la cui azione e dedizione furono alla base della vitalità e fedeltà al magistero teresiano delle case sorte nella penisola. ${ }^{3}$

Il fervore che la Congregazione di Sant'Elia manifesta in ordine all'impianto di nuove comunità di Teresiani è attestato dalla diffusione, traduzione e stampa

\footnotetext{
${ }^{1}$ Estato scritto che per Filippo II: «la presenza del preposito generale dell'ordine quale potere concorrente che risiede fuori dal paese è percepita come pericolo» Motta, F.- Pavone, S. 2005. «Per una storia comparativa degli ordini religiosi» Dimensioni e problemi della ricerca storica, $\mathrm{n} .1$, Identità religiose e identità nazionali in età moderna, M. Caffiero, F. Motta, S. Pavone (eds): 17. Cfr. Borromeo, A. 2003. «Filippo II e il Papato» in L. Lotti e R. Villani (eds), Filippo II e il Mediterraneo: 477-535. Roma-Bari: Laterza.

${ }^{2}$ Marchetti, E. 2005. «La riforma del Carmelo scalzo tra Spagna e Italia» in Identità religiose e identità nazionali: 61-80.

${ }^{3}$ Fra i primi Scalzi spagnoli, custodi dello spirito di Teresa, che collaborarono allo sviluppo della Congregazione d'Italia si ricordano: Pedro de la Madre de Dios (1565-1608) eletto anche preposito dell'Ordine e direttamente responsabile degli insediamenti romani: di Santa Maria della Scala, di San Giuseppe a Capo le Case, della prima casa di Napoli, e di San Silvestro a Montecompatri eretto in stretta collaborazione con il cardinale Baronio; Domingo de Jesús Maria (Ruzola) legato alle vicende di Santa Maria della Vittoria a Roma e a Praga, come anche alla fondazione di Santa Maria dei Rimedi a Palermo; Juan de Jesús Maria ed altri come lo scalzo p. Prospero del Espiritu Santo (Nalda, Logroño 1583- Monte Carmelo 1653) che entrò nel convento di Santa Maria della Scala (1607). Primo suo destino dopo il 1608 è Palermo, segue poi Napoli dove chiese, ed ottenne, di raggiungere il Deserto di Varazze da cui partirà per la missione in Persia e, in seguito, nei luoghi santi.
} 
delle opere della santa avilese nei territori italiani, ${ }^{4}$ dal rapido moltiplicarsi delle vocazioni e dalla mirata erezione di conventi. Nel 1600 la Congregazione d'Italia inaugurava, quindi, ufficialmente un cammino che già aveva avuto il suo inizio con il sorgere del primo convento maschile genovese di Sant'Anna nel 1584, seguito nel 1597 da quello romano di Santa Maria della Scala; ${ }^{5}$ un rapido ed efficace susseguirsi di fondazioni maschili, progressivamente approvate dal Capitolo Generale, che prosegue anche negli anni seguenti. ${ }^{6}$

Questo moltiplicarsi di conventi e, come vedremo, di monasteri femminili, attivò la rapida individuazione ed approvazione delle differenti Provincie scalze, la cui istituzione permetteva una coerente gestione delle nuove case e delle tante vocazioni. Nel Capitolo Generale del 12 maggio 1617, svoltosi nel convento di Santa Maria della Scala, furono istituite, per ordine di anzianità, le prime sei Provincie della Congregazione d'Italia: quella di Genova che includeva la Liguria, la Toscana, il Piemonte ed era formata da quattro conventi di frati e da un monastero di monache; quella di Roma - con il Lazio, l'Umbria, le Marche, il Regno di Napoli e la Sicilia - e quattro conventi di frati e un monastero di monache. Seguivano la Provincia di Polonia, costituita da tre conventi di frati e un monastero; quella della Lombardia estesa alle Venezie e alla Romagna e composta da quattro conventi di frati e dal monastero di Cremona legato, come vedremo, alla prima fondazione femminile in Italia, quella di Genova. ${ }^{7}$

\footnotetext{
${ }^{4}$ Marchetti, E. 2001, Le prime traduzioni italiane delle opere di Teresa di Gesù nel quadro dell'impegno papale post-tridentino, Bologna: Lo Scarabeo.

${ }^{5}$ Sulle vicende genovesi cfr. Roggero, A. 1984, Genova e gli inizi della Riforma Teresiana in Italia (1584-1597), Roma: Teresianum; Idem. 1996. «Origini della presenza carmelitana maschile e femminile a Genova», in S. Giordano -C. Paolocci (eds), Nicolò Doria. Itinerari economici, culturali e religiosi nei secoli XVI-XVII tra Spagna, Genova e l'Europa, II: 315-331. Roma: Teresianum. Sul quadro delle fondazioni romane si veda Ruzza di, O. 1987. Sintesi storico-cronologica della Provincia romana dei Padri Carmelitani Scalzi. Roma: Edizioni OCD.

${ }^{6}$ Questo l'elenco delle fondazione in cui compare anche la data di approvazione del Capitolo Generale, anche se in qualche caso l'effettiva erezione avvenne a distanza di parecchi anni dalla data di approvazione, come nel caso di Velletri e di Fermo: 1584: Genova (Sant'Anna); 1597: Roma (Santa Maria della Scala); 1602: Napoli (La Madre di Dio); 1605: Montecompatri; 1606: Cremona, Terni; 1607: Roma (Santa Maria della Vittoria); 1609: Loano; 1610: Palermo; 1611: Padula, Bologna; 1613: Fossombrone; 1614: Genova (Santa Maria della Sanità), Milano; 1616: Varazze; 1617: Faenza; 1619: Firenze, Baida, Velletri; 1620: Genova (San Carlo), Lecce; 1621: Pavia, Mondovì; 1622: Torino; 1623: Caprarola, Perugia, Parma; 1624: Napoli (Santa Teresa a Chiaia); 1626: Comiso; 1627: Piacenza; 1628: Cerra, Chiavari, Fermo, Portico, Savona. A questa lista, pochi anni dopo, si aggiunsero gli insediamenti che a partire dal 1677 costituiranno la Provincia Veneta dell'Ordine: 1633: Venezia; 1660: Brescia; 1664: Verona; 1669: Padova, Vicenza.

${ }^{7}$ Cfr. Fernández de Mendiola, D. 2011, El Carmelo teresiano en la historia. Una nueva forma de vida contemplativa y apostólica, Dos Congregaciones del Carmelo Descalzo. Desarrollo paralelo y visiones dispares (1597-1650), III: 354. Roma: Teresianum. Nello stesso Capitolo Generale furono approvate la Provincia di Francia, estesa anche alla Lorena (quattro conventi di frati e il monastero di Avignone); la Provincia delle Fiandre cui fu aggregato il convento di Colonia (sei conventi di frati e sei monasteri di Scalze). A parte restava il convento-missione di Ispahán in Persia (ibidem: 587-88).
}

Hispania Sacra, LXVII

136, julio-diciembre 2015, 467-503 ISSN: 0018-215X, doi: 10.3989/hs.2015.014 
Ma il ritmo con cui si approvarono le Provincie non si fermò: nell'ottavo Capitolo Generale del 1626 furono erette le due nuove Provincie: quella di Napoli (Regno di Napoli, Due Sicilie e isola di Malta), sotto il titolo della Madre di Dio, e quella tedesca intitolata al Santissimo Sacramento. Tuttavia già nel 1629 il Definitorio Generale stabilì che la Sicilia e Malta fossero governati da un Vicario provinciale, e solo tre anni più tardi, il Capitolo Generale del 1632, eresse la Sicilia a Provincia col titolo di Sant'Alberto. ${ }^{8}$ Inoltre prima dello scadere del secolo il Capitolo Generale celebrato a Roma dal 2 al 14 maggio 1653 eresse la Provincia del Piemonte con conventi separati da quelli di Genova; il Capitolo Generale del 1677 decretò la erezione della Provincia di Venezia, ${ }^{9}$ mentre il Capitolo Generale celebrato a Roma dal 22 aprile al 4 maggio 1695 eresse la nuova Provincia di Etruria con quattro conventi: quelli di Firenze, Siena a Arezzo e con la incorporazione provvisoria di quello di Urbino. Negli stessi anni si registra anche la erezione effimera della Provincia di Romagna nel tentativo fallito di una divisione dalla Provincia della Lombardia dei conventi di Bologna, Faenza, Ferrara e Forlì. Il XVII secolo - i primi cento anni dalla morte di Teresa - vede così completarsi e consolidarsi le case scalze dipendenti dalla Congregazione d'Italia.

\section{LE FONDAZIONI DELLE SCALZE}

È su questa trama di fondazioni e di sviluppi anche istituzionali che occorre proiettare il moltiplicarsi degli insediamenti femminili non soltanto all'interno della Congregazione italiana di sant'Elia, ma anche nei territori tedeschi e austriaci e negli Stati di Polonia e Francia. In quest'ultima, l'espansione auspicata e richiesta già dal 1585 a pochi anni dalla morte della santa, fu facilitata dal ristabilimento della pace religiosa tra cattolici ed ugonotti a seguito dell'Editto di Nantes del 1598, e dell'accordo firmato nello stesso anno tra Francia e Spagna con il Trattato di Vervins. Sorti in Francia prima delle case maschili, i monasteri

\footnotetext{
${ }^{8}$ La fondazione del primo convento teresiano in Sicilia risale al 1610 con Santa Maria dei Rimedi, mentre il primo monastero di monache carmelitane Scalze a Palermo è del 1627.

${ }^{9}$ Visto lo sviluppo degli insediamenti, il 21 aprile 1673 il Capitolo Provinciale di Lombardia accolse la richiesta dei padri di origine veneta di costituirsi in Provincia a motivo dei cinque conventi sorti su questo territorio (Venezia, Brescia, Verona, Padova, Vicenza). La domanda venne riportata al Capitolo Generale del 1674, che il 2 aprile staccò dalla Lombardia i conventi e li pose sotto la giurisdizione di un Commissario generale nella persona del padre Antonio Maria dell'Ascensione. Poco dopo, il Capitolo Generale celebrato nel 1677 creò la Provincia Veneta, eretta sotto il titolo di San Giovanni della Croce ed elesse il Provinciale e tutti i nuovi Superiori. Nel 1866 tutto fu bruscamente interrotto a motivo della soppressione attuata dal Governo italiano. Dopo la Seconda Guerra Mondiale, si ebbe il ritorno degli Scalzi in Sicilia quando, nel settembre del 1945, si affidò alla Provincia Veneta la restaurazione del Carmelo maschile in Sicilia. Il 13 agosto del 1946 il Padre Provinciale del Veneto accettò di dare inizio a tale restaurazione e chiese subito l'autorizzazione a fondare tre nuove case: Ragusa, Carlentini e Avola.
} 
di Teresiane godettero dell'appoggio di figure come quelle di Jean de Brétigny Quintanadueñas, ${ }^{10}$ Barbre Avrilllot Madame Acarie,,${ }^{11}$ Chaterine d'Orleans principessa di Longville, il più noto Pierre de Bérulle e di altri illustri religiosi, fra cui anche Francesco de Sales.

A differenza di ciò che, come si vedrà più oltre, avvenne in Italia, la Francia richiese tassativamente che per la prima fondazione femminile fossero impiegate monache provenienti dalla Spagna e che avessero ben conosciuto la stessa Teresa assimilando così alla fonte lo spirito della riforma. Nella prima casa sorta a Parigi giunserò così, nonostante la resistenza dei Superiori spagnoli, donne che avevano vissuto gli anni cruciali dell'inizio dell'avventura scalza: Ana de Jesús, ${ }^{12}$ Ana de san Bartolomé, infermiera e confidente di Teresa che morì tra le sue braccia, Isabel de los Angeles (Márquez) ${ }^{13}$ Beátriz de la Concepción. ${ }^{14}$

Le numerose e faticose trattative per impiantare in terra di Francia il Carmelo riformato furono affiancate dall'attivo intervento della curia romana, come emerge dagli studi su queste fondazioni, strettamente collegate alle case sorte in territorio fiammingo, sentite come pedine importanti dalla politica spagnola, impegnata ad incentivarle sui propri domini come strumento di coesione intorno al potere regio. ${ }^{15}$

${ }^{10}$ Juan de Quintadueñas-Brétigny (Rouen 1556-1634) conobbe lo scalzo Jerónimo Gracián nel 1581 a Siviglia e nel 1586 visitò la carmelitana Ana de Jesús nel monastero di Madrid. Ordinatosi sacerdote nel 1595, lavorò alla traduzione francese degli scritti di Teresa che venne pubblicata nel 1600 . Convinto sostenitore della iniziativa Scalza, contribuì attivamente alla fondazione del primo monastero di Scalze a Parigi nel 1604. Cfr. De Serouet, P. 1974, Jean de Brétigny, aux origines du Carmel de France, de Belgique et du Congo, Louvain.

${ }^{11}$ Madame de Acarie, parente di Pierre de Bérulle si fece carico degli aspetti materiali relativi all'arrivo delle Scalze a Parigi. In casa sua si riunivano le «Dévotes», cioè dame appartenenti alla aristocrazia francese desiderose di propagare, anche per mezzo di attività catechetiche, il nuovo cattolicesimo trionfante. In seguito Barbre Avrillot Acarie entrò nel neofondato monastero scalzo parigino con il nome di Maria dell'Incarnazione. Cfr. Rapley, E. 1990. The 'Dévotes'. Women and Church in Seventeenth Century France, MC Gill-Queen's University Press Montreal \& Kingston.

${ }^{12}$ Ana de Jesús fu discepola e collaboratrice di Teresa insieme alla quale fondò, nel 1575, il monastero di Beas di cui fu priora. In seguito diede inizio ai monasteri di Granada (1582) e Madrid (1586). Nel 1604 introdusse la Riforma teresiana in Francia, dove fondò numerosi monasteri per poi passare nel 1607 in Belgio dove promosse il monastero di Bruxelles.

${ }^{13}$ Nata a Villacastín nel 1565, nel 1591 emise la professione nel monastero di Salamanca. Fu tra le prime fondatrici in Francia dove rimarrà fino alla morte avvenuta nel 1644.

${ }^{14}$ Nasce nel 1569 ad Arévalo dalla famiglia degli Zuñiga e nel 1593 professa nel monastero di Salamanca. Fu compagna di Ana de Jesús nella fondazione di Bruxelles di cui fu anche priora. Nel 1630 rientrò in Spagna nello stesso convento di Salamanca dove esercitò la funzione di priora. Morì nel 1646.

${ }^{15} \mathrm{Un}$ atteggiamento analogo è riscontrabile, un secolo dopo l'inizio della Riforma teresiana, anche presso Ferdinando III e la corte viennese come hanno riscontrato studi recenti di Chiara Ghezzi, La Madonna delle Laste a Trento nel XVII secolo. Vita religiosa e aspetti artistici di una fondazione dei Carmelitani scalzi. Tesi di Laurea sotto la direzione della prof.ssa Elisabetta Marchetti, Università di Bologna Facoltà di Conservazione dei Beni culturali, A.A. 2006-2007.

Hispania Sacra, LXVII

136, julio-diciembre 2015, 467-503 ISSN: 0018-215X, doi: 10.3989/hs.2015.014 
Diversa la situazione per l'Italia dove le uniche fondatrici venute dalla Spagna furono quelle responsabili dell'insediamento genovese. Qui, nel 1590 - precedute dallo stanziamento degli Scalzi che fondarono il convento di S. Anna nel 1584, secondo uno schema che si ripete con regolarità - giunsero le Teresiane. Scrive Roggero:

Tre delle cinque fondatrici spagnole che furono al monastero di Gesù Maria di Genova faranno giungere il loro ricordo della Madre Teresa deponendo ai processi di beatificazione: la madre Geronima dello Spirito Santo, in quelli istituiti a Madrid nel 1595 dove era priora dopo il ritorno da Genova; le altre due, Marcella di S. Giuseppe e Maria di S. Gerolamo, ancora nel 1595, in quello istituito a Malagón dove erano ritornate, perché loro monastero di origine. Le dichiarazioni che ci sono conservate danno una idea di quanto fosse pregno di teresianesimo il monastero di Genova che le testimoni avevano lasciato appena da un anno e nel quale avevano fatto rivivere i loro ricordi e relazioni con la Madre Teresa. ${ }^{16}$

Ma queste Scalze, a cui si era unita per la fondazione Maddalena Centurione, vennero richiamate dai superiori spagnoli già dal 1594 in Spagna: una decisione, radicalmente opposta alla politica adottata dal precedente generale, il p. Nicolò Doria, che marca chiaramente un cambio di atteggiamento da parte dell'Ordine in relazione alla volontà e all'aiuto per la espansione in Italia. Da questo momento i superiori spagnoli abbandoneranno l'attenzione verso le case italiane e - in primis - verso quelle femminili. Del gruppo delle monache giunte a Genova dalla Spagna rimarrà nel capoluogo ligure, divenendo subito priora del monastero, solo Maddalena Centurione, la promotrice e prima finanziatrice dell'impresa genovese. ${ }^{17}$

Il monastero di Gesù e Maria, insieme alla fondazione maschile di Sant'Anna, si radica ben presto nella città ligure con l'approvazione di una parte significativa del patriziato genovese, le cui figlie faranno a gara per entrare nel numero ristretto delle teresiane, fissato a venti dalle Costituzioni del 1582. Dal 1591 al 1599 sono infatti ammesse nel monastero ventuno professioni solenni, espresse da numerose

${ }^{16}$ Roggero, A. 1984: 121. Si veda inoltre il manoscritto Libro de la Fundación y Professiones de este monasterio de Jesús Maria de Carmelitas Descalças de la ciudad de Génova, Savona, Archivio Monasteto di Santa Teresa (AMST), ms.2, f. 13ss.

${ }^{17}$ Maddalena Centurione, moglie di Agostino Spinola, si trovava in Spagna negli anni che videro concretarsi a Genova la fondazione del primo convento maschile di Sant'Anna (1584). In Spagna conobbe alcuni Scalzi tra cui Jéronimo Gracián e Nicolò Doria. Rimasta vedova nel 1589 la Centurione, volendo attuare un suo antico desiderio di entrare in religione, si rivolse al Doria che le propose di realizzare il suo progetto a Genova dove era stata richiesta una fondazione. Ottenuto nell'agosto del 1590 da Roma, e per mezzo del cardinal protettore dell'Ordine Domenico Pinelli, il permesso di erigere un nuovo monastero, il Doria designò le monache fondatrici provenienti tutte dal monastero di Malagón. Il viaggio verso la città ligure iniziò il 28 settembre 1590 e subì una sosta di tre mesi a Barcellona dove Maddalena Centurione ricevette 1'abito dalla futura priora di Genova, la madre Geronima dello Spirito Santo. Ripreso il viaggio il 12 dicembre le Scalze giunsero a Genova e si stabilirono provvisoriamente nel monastero di San Gerolamo al Roso, infine il 7 febbraio 1594 venne inaugurato il nuovo monastero di Gesù Maria. 
rappresentati di casati nobiliari come le due Centurione, una Malaspina figlia del Marchese di Fosdinivo, che sarà seguita in monastero dalla madre Livia Doria. Non solo è rappresentata la nobiltà genovese, ma il monastero di Gesù Maria richiama aspiranti anche da Milano: la figlia del marchese di Soncino Isabella Stampa, e la cugina Ottavia Pietra, figlia del conte Pietro di Milano. Varie sono le Scorza e le due sorelle Angela e Brigida Sauli, figlie di Anfrano Sauli. ${ }^{18}$

Nel primo volume della traduzione della Riforma stampato a Genova nel $1654,{ }^{19}$ il p. Gasparo di san Michele, traduttore dell'opera, dedica il volume alle «Carmelitane Scalze del venerabil Monastero di Gesù Maria di Genova», soffermandosi sul diretto appoggio di una parte ben identificata delle famiglie genovesi e sul ruolo di questo insediamento. Sottolineando, nella lettera dedicatoria, il forte rapporto che la casa genovese mantiene con la Madre Teresa grazie alle fondatrici spagnole che era vissute in intimità con lei, il traduttore ricorda che da Genova ebbe inizio la catena dei carmeli femminili italiani, fatta eccezione per «l'anomalo» caso romano, di cui si parlerà più oltre.

Diversamente dalle monache spagnole, richiamate in patria dai superiori, le Scalze genovesi non lasceranno i conventi da loro fondati per tornare al monastero di origine; e ciò anche se andranno in terre straniere. Ma questo percorso di fondazione, partito da Genova, manca a tutt'oggi - tranne pochi casi, come quelli di Genova, Roma e dei Deserti e santuari scalzi $-{ }^{20}$ di ricerche puntuali che diano conte del forte radicamento in Italia della lezione di Teresa. ${ }^{21}$ Non si dispone inoltre di elementi sufficienti per individuare con certezza secondo quale criterio o disegno vennero eletti i luoghi accettati dalle genovesi come nuove case, anche se è possibile confermare che di norma le Teresiane si stanziavano in città in cui era già presente il ramo maschile e in cui vi fossero richieste esplicite di fondazione e il concreto aiuto di facoltosi e influenti personaggi o famiglie. ${ }^{22}$

\footnotetext{
${ }^{18}$ Roggero, A. 1984:139.

${ }^{19}$ Francesco di Santa Maria, Riforma de’ Scalzi di Nostra Signora del Carmine dell'Osservanza primitiva fatta da Santa Teresa di Giesu, vol. I trad. di Gasparo di San Michele, Genova 1654; vol. II trad. Girolamo di Santa Teresa, Bologna 1662.

${ }^{20}$ Cfr. Sturm, S. 2002. L'eremo di Montevirginio e la tipologia del Santo Deserto, Roma: Gangemi Editore. Per il tema dei santuari legati alla storia dell'Ordine si veda Marchetti, E. 2013. «L'Ordine dei Carmelitani scalzi e i Santuari tra XVI e XVIII secolo», in L.M. Olivieri (ed), Ordini religiosi e santuari in età moderna e contemporanea: 207-221. Bari: Edipuglia.

${ }^{21} \mathrm{Si}$ veda la panoramica proposta da Mejia Maya, R. 1995. Las fundaciones de las Camelitas Descalzas en Italia, Malta y paises balcanicos (1590-1992). Carmelos en el mundo. Burgos: Editorial Monte Carmelo.

${ }^{22}$ Per l'analisi delle politiche e le strategie di espansione del ramo maschile in Italia si veda il volume La chiesa di Santa Maria di Nazareth e la spiritualità dei Carmelitani Scalzi a Venezia, 2014. G. Bettini e M. Frank (eds), Venezia: Marcianum Press e in particolare: Raines, D. La lobby cittadina dei Carmelitani Scalzi nella Venezia settecentesca: 77-100 e Marchetti, E. Venezia e Santa Maria di Nazareth: tappe significative nello sviluppo culturale e nella diffusione della realtà scalza: 101-112.
}

Hispania Sacra, LXVII

136, julio-diciembre 2015, 467-503 ISSN: 0018-215X, doi: 10.3989/hs.2015.014 
La prima filiazione del monastero genovese fu, nel 1607, Napoli dove sotto la guida di Maddalena Centurione, già fondatrice e priora a Genova, sorgerà il monastero di San Giuseppe; da qui le Teresiane saranno poi introdotte nel 1627 a Palermo dalla madre Dorotea Mercante. Dalla capitale del Regno di Napoli le Scalze giungeranno inoltre a Terni, guidate dalle madri Caterina Pietra e la cugina Teresa Stampa. La seconda fondazione dipendente direttamente da Genova fu quella di Cremona primo monastero della Provincia Lombarda. Partite da Genova il 25 marzo del 1613 le fondatrici poterono assistere il 5 aprile alla inaugurazione del monastero, alla messa celebrata dal vescovo Brivio, alla riposizione del Santissimo Sacramento e alla instaurazione della clausura. In questo primo gruppo compare la madre Maria di Gesù (Castagnoli) che, nel 1619, passerà a fondare a Bologna e Maria di San Alberto che, nel 1635, andrà a fondare a Parma.

Anche a Bologna l'arrivo degli Scalzi precede di qualche anno e prepara il giungere delle monache. Nell'ottobre 1611 infatti i primi padri entrarono a Bologna: ospiti in un primo tempo dei Calzati della Congregazione di Mantova in San Martino, pochi giorni dopo presero in affitto una casa di fronte alla chiesa parrocchiale di San Giuliano. Con la mediazione del cardinal Borghese, arcivescovo di Bologna, e del conte Orazio Ludovisi, e con il sostegno dello scalzo Domingo de Jesús María, futuro venerabile ${ }^{23}$ ottennero nel 1618 di poter officiare nella chiesa di Santa Maria Lagrimosa che, ceduta nel 1619, venne definitivamente occupata dai Teresiani nel 1622.

Intorno a questa chiesa e ai padri crebbero negli anni le testimonianze di sostegno ed apprezzamento da parte dei bolognesi e, in particolare, della nobiltà, come nel caso dell'appoggio prestato dal cardinale Bernardino Spada, legato di Urbano VIII, e dal cardinal Borghese. ${ }^{24}$ Quanto alla fondazione bolognese delle Teresiane, i principali benefattori e protettori furono i fratelli Marcantonio e Flaminio Campana che, comprato un locale in via Santo Stefano di fronte alla chiesa di San Giuliano, lo adattarono come monastero. Superata l'iniziale ritrosia della città ad accogliere un ulteriore monastero sul suo territorio, le Scalze seppero conquistare l'appoggio delle famiglie più in vista - i Ludovici, gli Orsi - mentre fu il senatore Bianchetti ${ }^{25}$ ad ottenere da Paolo V il breve di fondazione. Da Cremona giunsero allora le quattro monache fondatrici, madre Maria di Gesù, madre Maddalena del SS. Sacramento, madre Francesca di S. Angelo e la conversa suor Maria Caterina di S. Onofrio; il primo novembre del 1619 la chiesa

\footnotetext{
${ }^{23}$ Cfr. Giordano, S. 1991. Domenico di Gesù Maria Ruzola (1559-1630). Un carmelitano scalzo tra politica e riforma nella chiesa posttridentina, Roma: Teresianum.

${ }^{24}$ Cfr. Bologna, Archivio di Stato (ASB), Corporazioni religiose (Fondo Demaniale) I. B, S. Maria Lacrimosa agli Alemanni, serie 7/6974. Soppresso nel 1797 il convento riaprì nel 1933 sempre in Via Santo Stefano, ma nella chiesetta del monastero di S. Omobono un tempo delle Scalze

${ }^{25}$ Cfr. Dolfi, P.S. 1990. Cronologia delle famiglie nobili di Bologna. Bologna: Ed. Forni.
} 
del monastero fu dedicata a San Gabriele Arcangelo. ${ }^{26}$ Nel 1637 le monache lasciarono questa prima casa per trasferirsi nel monastero di sant'Omobono fabbricato ex novo. ${ }^{27}$

Tra la documentazione relativa al monastero bolognese è conservato il Ristretto della Vita della Madre Maria Valeria della Croce Carmelitana Scalza priora del Convento delle R.R. Madri scalze di San Gabriele a Bologna ${ }^{28}$ in cui si narra la vita della religiosa, appartenente al nobile casato degli Orsi, che il 3 febbraio 1623 professò nel monastero. Il testo ripercorre la vita della monaca soffermandosi su momenti e circostanze analoghi a quelli vissuti dalla stessa Teresa che rimane modello e meta di imitazione, nonché inveramento sia della santità della scalza sia della esemplarità della casa che la accolse. Ecco allora descritta l'iniziale avversione della giovane per la vita monastica, successivamente abbracciata nonostante la opposizione dei familiari. Molti e significativi i punti di contatto tra la vita di Maria Valeria e la santa fondatrice con la quale la priora bolognese sembra confondersi: la grave infermità sopportata dalla giovane, il ricorso fiducioso alla orazione, il ruolo di guida, materna e forte, nei confronti delle consorelle e, soprattutto, la delicata e totale obbedienza a superiori e confessori.

Oltre a farsi carico, nel 1613, della fondazione del monastero di Avignone, da cui dipenderanno poi le case di Carpentras e Chambery, le Teresiane genovesi del Gesù e Maria promossero, nel 1619, la nascita del secondo monastero di Scalze sorto nella città, al quale contribuì la made Geronima Centurione che, senza posa, passò poi come fondatrice a Firenze. Nel 1623 le genovesi diedero poi inizio al monastero di Santa Teresa a Savona, al quale seguì quello dello Spirito Santo nella stessa città. In totale le fondazioni scaturite dal primo monastero di Genova furono sei, e compresero anche il monastero Vienna, da cui sarebbe sorto quello di Graz. La seconda metà del XVII secolo registra, accanto al notevole consolidarsi delle case esistenti, una ulteriore espansione. Vennero infatti eretti sotto la giurisdizione dell'Ordine i seguenti monasteri: Modena (1652), Savona (1653), Regina Coeli (1654), Mantova (1660), Piacenza (1673), Milano (1674), Conegliano (1682); Messina (1686); Casalnocetto (1687); Reggio Emilia (1689), Marsala (1691), Milazzo (1691), Corleone (1692), Guastalla (1693); Arezzo (1694); Ivrea (1695), San Giorgio di Venezia (1697). Ossia 17 monasteri femminili a fronte di 40 conventi maschili sorti negli stessi anni.

\footnotetext{
${ }^{26}$ ASB, Corporazioni religiose (Fondo Demaniale) I. A, M.M. Scalze di S. Gabriele di Bologna, serie: $1 / 1861 ; 36 / 1896$.

${ }^{27}$ Cfr. Fini, M. 2007. Bologna sacra, Bologna: Pendragon: 75 e 173.

${ }^{28}$ Biblioteca Comunale dell'Archiginnasio di Bologna (BCABO), Ristretto della Vita della Madre Maria Valeria della Croce Carmelitana Scalza priora del Convento delle R.R. Madri scalze di San Gabriele a Bologna, B. 1671.
} 
Un accenno particolare va riservato alla fondazione del monastero romano di San Giuseppe a Capo le Case, sorto nel 1598 e definito 'atipico' per l'assenza di fondatrici scalze, dovuta al mancato invio di monache da Genova, e per il totale disinteresse da parte dei superiori spagnoli, decisi - come si è precedentemente detto - a far ritornare le fondatrici in patria. La casa romana venne quindi eretta per volontà di Clemente VIII il quale stabilì che essa rimanesse sotto la giurisdizione del Vicariato di Roma esercitata - sempre per indicazione pontificia dall'oratoriano cardinal Cesare Baronio. ${ }^{29}$

A Roma come a Genova, dunque, le prime fondazioni italiane di Teresiane furono sorrette dagli aiuti e cure da parte di una generazione di Scalzi e da personaggi legati al Carmelo da rapporti di fiducia ed ammirazione, fra cui si ricordano il p. Jéronimo Gracián - espulso dagli Scalzi ma fervente promotore di insediamenti carmelitani $-^{30}$ e gli illustri oratoriani Filippo Neri, Cesare Baronio, Francesco Soto. ${ }^{31}$

${ }^{29}$ Breve Relatione delle Cose più notabili havenute nel ven.le monasterio di san Gioseppe delle Carmelitane Scalze del instituto della seraff.ca M.re Santa Teresa di Giesù in San Gioseppe a Capo le Case, Roma, Archivio Generale presso la Curia Generalizia OCD (AGOCD), Plut.88 E. Baronio è presente nella vita del monastero romano femminili di San Giuseppe a Capo le Case quale responsabile della guida spirituale di alcune delle prime Scalze italiane: Clemente VIII, inoltre, nel 1587 assoggettò questa fondazione al cardinale oratoriano la cui autorevolezza e protezione servirono efficacemente anche per facilitare la diffusione delle opere tradotte di Teresa minacciate da una lunga vicenda inquisitoriale. Anche l'insediamento carmelitano di San Silvestro a Montecompatri sembra aver beneficiato dell'intervento del Baronio che, del resto, fu amico e collaboratore sia del padre Gracián (1584-1614) - braccio destro di Teresa di Gesù e delle sue iniziative - sia di Pedro de la Madre de Dios (1565-1608) e dei primi Carmelitani stabilitisi a Roma a Santa Maria della Scala. Sulla 'atipicità' della fondazione romana, pur nella completa fedeltà all'insegnamento della Santa, si veda Marchetti E, 2001; Eadem, 2000. «Il Carmelo scalzo e gli oratoriani a Roma» Archivio della Società di storia patria, 123: 105-131.

${ }^{30}$ Jéronimo Gracián de la Madre de Dios (1545-1614) prese nel 1572 l'abito degli Scalzi a Pastrana. Ben presto Teresa vide nel carmelitano un valido aiuto per la promozione e difesa della sua iniziativa. Gracián venne eletto come primo Provinciale degli Scalzi, ma dopo la morte della santa si manifestarono opposizioni e critiche contro di lui e il suo governo. Venne sostituito nella carica dal p. Nicolò Doria con il quale entrò in conflitto. Espulso dall'Ordine il p. Jéronimo si recò a Roma dove non riuscì ad entrare nelle file degli Scalzi della Congregazione italiana. Dopo essere stato prigioniero dei turchi, ritornò a Roma dove svolse una intensa attività, in seguito venne ammesso tra i Carmelitani calzati. Si recò poi in Spagna e nelle Fiandre dove morì.

${ }^{31}$ Francesco Soto de Langa (Osma 1536-Roma 1619) ammesso giovanissimo alla Cappella Pontificia, senza mai abbandonare questo servizio, entrò nella cerchia di Filippo Neri. Le sue abilità canore e musicali contribuirono notevolmente all'affermarsi e allo sviluppo di quella particolare modalità apostolica propria dei filippini denominata «oratorio». Aringhi, P. 1885. «Vita inedita del Padre Francesco Soto» San Filippo Neri, gennaio-febbraio: 7-8; marzo-aprile: 4-6. Il manoscritto dell'opera in Biblioteca Vallicelliana, ms O. 58, Le vite e detti di padri, e fratelli della Congreg.ne dell'Oratorio di S. Filippo Neri fondata nella Chiesa di S.Maria in Vallicella. Raccolti da Paolo Aringhi della detta Congregatione e da altri, I. 
La crescita e i cambiamenti istituzionali che coinvolsero il Carmelo scalzo nel primo secolo della sua vita riguardarono anche la normativa costituzionale alla quale l'Ordine affidava, per una parte rilevante, il compito di stabilire regole condivise per le numerose comunità teresiane in continua espansione. Riassume efficacemente Eulogio Pacho:

Entre 1562-67 y 1607, se registran siete textos constitucionales para las Carmelitas Descalzas: cinco en español y dos traduciones, una italiana y una francesa. Su aparición estuvo motivada por los cambios introducidos progresivamente en el gobierno de las religiosas. Sucedieron por este orden: en primer lugar, el texto primitivo de San José de Avila, redactado entre 1562 y 67, revisado y aprobado este mismo año por el general Juan Bautista Rossi o Rubeo en 1567; le sucedió el preparado y promulgado por el Capítulo de Alcalá de 1581; éste fue sustituido por el que promulgó el papa Sisto V, en 1590; a su vez, éste fu revisado por Gregorio XIV y publicado en 1592. El texto constitucional de 1592 aparecía en italiano en 1593, mientras la version francesa de las Constituciones de 1581 aparecía en $1607 .{ }^{32}$

La genesi, le cause della evoluzione e i protagonisti che parteciparono alle diverse redazioni delle costituzioni teresiane sono stati oggetto di analisi da parte della recente storiografia dell'Ordine che, interpretando le proprie antiche fonti, ha messo in evidenza le diverse posizioni che manifestarono le Scalze francesi e quelle italiane in merito al testo fondante del Carmelo teresiano, segno di un dibattito che si svolse in seno al Carmelo fra gli ultimi anni del Cinquecento e il primo trentennio del Seicento, e che qui si vuole brevemente ripercorrere. ${ }^{33}$

Nel 1593 a Genova venivano date alle stampe le Regola e Costitutioni delle religiose primitive dell'Ordine della Gloriosa Vergine Maria del monte Carmelo: ${ }^{34}$ un testo che traduceva fedelmente dallo spagnolo il testo costituzionale del 1592, emanato da Gregorio XIV, accettato dalla prima comunità genovese e trasmesso alle successive case che da essa dipesero, e per questo divenuto la legislazione predominante per i monasteri posti sotto la giurisdizione della Congregazione italiana. ${ }^{35}$

${ }^{32}$ Constituciones de las Carmelitas Descalzas (1562-1607). 1995: XII. Edición preparada por T. Alvarez, A. Fortes, F. Antolín, S. Giordano, S.M. Morgain, B. Velasco, Roma: Teresianum.

${ }^{33}$ Fortes, A. OCD. 1988. «Textos constitucionales de las Carmelitas Descalzas» Monte Carmelo 96: 509-550; 97(1989): 88-125 e 483-518; 99(1991): 335-363. Silverio di S. Teresa OCD, «Constituciones primitivas», in Biblioteca Mistica Carmelitana (BCM) 6: VII-XXX e, soprattutto, Constituciones de las Carmelitas Descalzas. 1995.

${ }^{34}$ Regola e Costitutioni delle religiose primitive dell'Ordine della Gloriosa Vergine Maria del monte Carmelo, In Genoua, per gli Heredi di Gir. Bartoli, MDXCIII.

${ }^{35}$ Cfr. Giordano, S. 1995, Costituzioni delle Carmelitane scalze d'Italia, 1593 in Constituciones de las Carmelitas Descalzas: 1995: 201.

Hispania Sacra, LXVII

136, julio-diciembre 2015, 467-503 ISSN: 0018-215X, doi: 10.3989/hs.2015.014 
Il percorso dell'edizione italiana del 1593 si arricchisce di una tappa ulteriore nel 1630, quando lo scritto venne rieditato dal preposito generale Ferdinando di santa Maria in una versione rivista in cui la modifica di maggior rilievo riguardava la gerarchia dei superiori; per le monache, in particolare, si stabiliva che esse erano «soggette al Padre Generale delli Carmelitani Scalzi della Primitiva Regola, e che il detto Padre Generale le possa visitare da per sé o per il Visitatore che vorrà nominare». ${ }^{36}$

Le Costituzioni del 1593, e la loro revisione del 1630, avevano comunque alle spalle una storia tormentata. Il testo costituzionale del 1592 era stato infatti frutto, all'interno dell'Ordine, di un forte dibattito che aveva coinvolto la stessa Monarchia e le cui origini risalivano al capitolo tenutosi a Pastrana nel 1585. In questa occasione Nicolò Doria aveva ottenuto nuove misure di governo, rivolte ad aiutare il provinciale nel disimpegno del suo ufficio. Era stato dunque varato un nuovo organo di governo, la Consulta, che trasferiva «l'autorità personale del provinciale ai suoi consiglieri o definitori tra i quali ci sarebbe stato un primus inter pares»»». ${ }^{37}$ Successivamente, nel 1587, Doria ottenne anche il breve papale Cum de Statu con cui si introdusse definitivamente nell'Ordine la Consulta, percepita però da Gracián e da alcune Scalze contraria allo spirito della Madre Teresa. Di fatto, «la institución de la Consulta supuso un cambio exorbitante en el régime de la vita de los Carmelitas descalzos y reclamó una alteración de las Constituciones para que la ley y la practica no estuviesen disociadas». ${ }^{38}$

Per quanto riguardava le monache, la Consulta interveniva in molti aspetti della vita regolare - nell'elezione delle priore, nella libera scelta dei confessori e le lamentele delle Scalze non mancarono:

Lo primero porque les obligaban a que de sus menudencias y defectillos ordinarios se diese noticia a siete personas (...) Lo segundo porque casi les quitaban las eleciones de las prioras, y las hacía muchas veces la Consulta, como más le parecía convenir, que era quitarles la libertad en ellas. Lo tercero porque frequentemente se hacían informaciones jurídicas de casos menudos, y como las monjas son temorosa de Dios, sencillas y ignorantes de lo que deben decir o callar facilísimamente se turban. ${ }^{39}$

Nonostante le assicurazioni che in nulla sarebbe mutato quanto disposto da Teresa per il governo, il 5 luglio 1588 l'Ordine approvò il testo Acta para Nuetras Hermanas ${ }^{40}$ in cui si dichiarava che le monache, come i frati, dove-

\footnotetext{
${ }^{36}$ Constitutioni 1630 , cap. I

${ }^{37}$ Roggero, A. 1984: 32.

${ }^{38}$ Sierra, T. 1996. Nicolás Doria, innovador del gobierno de la Reforma carmelitana, in S. Giordano C. Paolocci 1996: 224.

${ }^{39}$ Reforma de los Descalzos de Nuetra Señora del Carmen, II, Madrid, 1655: 431-432.

${ }^{40}$ Monumenta Historica Carmeli Teresiani (MHCT), III, doc. 349: 314-315. Cfr. Moriones, I. Ana de Jesús y la herencia teresiana, ¿Humanismo cristiano o rigor primitivo? Roma: Teresianum.
} 
vano essere sottoposte al governo della Consulta la quale avrebbe assegnato i confessori, il procuratore e, a suo tempo, i visitatori. Il malcontento si concretò, allora, nell'invio di libelli a Filippo II; e se, da un canto, Doria era impegnato a salvaguardare il proprio punto di vista, dall'altro la schiera di sostenitori del Gracián non rimase inattiva. Questi ultimi ottennero l'appoggio di Luis de León e di alcune tra le prime carmelitane che, come Ana de Jesús, decisero di ricorre a Roma perché fossero salvaguardate le stesse Costituzioni.

Come frutto di tali azioni il 5 giugno 1590 Sisto V concedeva il breve Salvatoris Nostri che: «approva le costituzioni della Madre Teresa e contiene una revoca con valore retroattivo, riguardante eventuali mutazioni fatte dalla legittima autorità che, d'ora innanzi, dovrà lasciare le costituzioni inalterate. $\mathrm{Si}$ istituisce un commissario generale che deve essere eletto nel capitolo degli Scalzi e aver posto subito dopo il vicario generale. A lui venivano affidati le visite e il governo delle monache con piena giurisdizione e potere senza che la Consulta, i provinciali e gli ordinari del luogo vi si potessero intromettere». ${ }^{41}$ L'intervento papale depotenziava il potere della Consulta in relazione al governo ordinario delle Scalze, privandola dell'influenza legislativa nel campo delle costituzioni. Contando allora sull'appoggio del re e dei superiori dell'Ordine, Doria ottenne il 25 aprile 1591 il contro breve Quoniam non ignoramus con il quale il papa: «trata de moderar las diferencia entre los dos grupos tanto en cuanto al gobierno de la Consulta, come en cuanto a las Constituciones de las Monjas». ${ }^{42}$ Sarà, infine, questo l'ultimo testo, frutto di una laboriosa mediazione, quello che il monastero di Genova accetterà e passerà alla maggior parte delle sue fondazioni.

Le cose andarono diversamente nel Carmelo francese dove le case femminili adottarono il testo costituzionale spagnolo del $1588,{ }^{43} \mathrm{e}$ non quello del 1592. La scelta evidenzia il lungo braccio di ferro che oppose gli Scalzi a Bérulle, con la vittoria di quest'ultimo, ostile all'applicazione in Francia del primo articolo delle Costituzioni di Alcalà (1581) che affidavano il governo delle Scalze al Carmelo.

Ancora una volta l'attenzione era focalizzata sul governo del ramo femminile dell'Ordine. Le monache che vennero inviate come fondatrici a Parigi avevano

\footnotetext{
${ }^{41}$ Roggero, A. 1984: 101.

${ }^{42}$ Fortes, A. Constituciones de Sixto V-Gregorio XIV 1592, in Constituciones de las Carmelitas Descalzas. 1995: 154.

${ }^{43}$ Morgain, S.M. 1995. Pierre de Bérulle et les Carmélites en France, La querelle du gouvernament, 1583-1629, Paris. Sérouet, P. 1974. «Jean de Brétigny (1556-1634). Aux origine du Carmel de France, de Belgique et du Congo» Bibliothèque de la Revue d'Histoire Ecclésiastique, 60. «Quintanadueñas. Lettres de Jean de Brétigny (1556-1634)», 1971, P. Sérouet (ed), Bibliothèque de la Revue d'Histoire Ecclésiastique, 53, Louvain.
} 
partecipato, in Spagna, al contrasto svolto a salvaguardare le Costituzioni teresiane del 1588; giunte poi in territorio francese intesero continuare a preservar e far conoscere il genuino spirito teresiano. La bolla papale In Supremo, del 13 novembre 1603, tra i principi enunciati stabiliva che il governo e la amministrazione del monastero di Parigi, prima casa scalza in Francia, fossero affidati a tre ecclesiastici: Jacques Gallemant (1559-1630), André Duval (1564-1638) e Pierre de Bérulle. Ana de Jesús si trovò presto in contrasto con tali disposizioni che prevedevano che esistesse una differenza gerarchica tra i vari monasteri e che le monache fossero governate da tre ecclesiastici. Tra questi vi era Pierre de Bérulle che, conoscendo le difficoltà esistenti in Spagna tra Scalzi e Scalze in relazione alle stesse costituzioni e alla forma di governo, era propenso ad evitare alle monache spagnole in Francia contatti con i padri dell'Ordine. Tuttavia la bolla del 1603 lasciava prudentemente spazio alla possibilità di modifiche dopo che fosse avvenuta in territorio francese la fondazione di un convento di Scalzi. A motivo di queste complesse e vicende ed influenze, ${ }^{44}$ il Carmelo francese mutuò tendenze e devozioni legate alla figura del Bérulle come, ad esempio, la devozione per l'Incarnazione, per santa Maria Maddalena e il controverso voto di schiavitù. Ai superiori spagnoli dell'Ordine parve illegittima la giurisdizione delle monache instaurata in Francia; Bérullle però poteva contare sull'appoggio del re di Francia, del papa e dei superiori carmelitani italiani. In parte per questi contrasti e nel timore di non poter tutelare adeguatamente le Costituzioni teresiane, alcune madri fondatrici spagnole decisero di lasciare la Francia per portare le fondazioni nelle Fiandre come fece Ana de san Bartolomé che «tornata a Parigi (1611), ottenne di passare in Fiandra per porsi sotto la direzione dei Carmelitani Scalzi, nonostante 1'opposizione del Bérulle». ${ }^{45}$

Lo sviluppo e il dibattito sulle Costituzioni e sul governo delle Scalze - processi condizionati dai più generali cambiamenti istituzionali dell'Ordine e da concrete vicende politiche inerenti alle nazioni che in quegli anni accolsero la espansione fondazionale dell'Ordine - costituiscono ancora un rilevante campo di riflessione ed osservazione su come il lascito di Teresa venne interpretato, vissuto e trasmesso e su quanto il ramo femminile e il suo governo venisse sentito quale snodo nevralgico da gran parte degli attori coevi: Ordine, Monarchia e Papato.

${ }^{44}$ Risulta emblema di questa complessità quanto affermato nello stesso Dizionario Carmelitano: «Con la diffusione dei monasteri delle Carmelitane Scalze in Francia, si ebbe nel 1607 a Bruxelles la pubblicazione della traduzione in francese delle Costituzioni di Alcalá (1581) con le modifiche del 1588. Risulta difficile comprendere perché non sia stato assunto il testo del 1592. Queste costituzioni in francese vennero ristampate nel 1613,1616,1620,1626, 1634 e in Belgio e in Polonia furono osservate almeno in alcuni monasteri che non vollero osservare il testo doriano» in Dizionario carmelitano, diretto da Boaga, E. O. Carm - Borriello, L. OCD, Roma: Città Nuova: 201.

${ }^{45}$ Macca, V. 2008. «Anna di S.Bartolomeo (Anna Garcia) beata, monaca OCD (1549-1626)», in Dizionario carmelitano. 2008: 31. 
Le teresiane nella Palermo sPagnola

Protagoniste delle prossime pagine saranno le prime due fondazioni di Scalze sorte nella capitale del Viceregno siciliano, scelte come studio di caso perché le relazioni del dominio italiano degli Austrias con la corte madrilena - esemplificate dalla presenza del viceré, dalle molte illustri famiglie d'origine ispanica residenti nell'isola e dai tanti «maritaggi» che legarono uomini e donne nati nei due paesi $-{ }^{46}$ rendono particolarmente interessante questo segmento della storia delle figlie di Teresa d'Avila nel territorio italiano. Una storia che si svolse in quella società eclesizada, permeata di elementi religiosi, che dette vita ad un «universo ordinado de conductas, sensibilidades y justificationes» al quale parteciparono tutti gli individui la cui identità cattolica si esprimeva anche nel rispetto della gerarchia politica e sociale e dell'ordine stabilito. ${ }^{47}$

Come per la maggior parte delle fondazioni sorte negli antichi Stati italiani, anche quelle che si dislocarono nell'isola nel corso del XVII secolo godettero della protezione e della munificenza di esponenti dell'aristocrazia: come ad esempio i monasteri sorti nelle città di Palermo, Messina e Catania, che ebbero come fondatrici donne appartenenti a dinastie prestigiose il cui segmento siciliano di storia si intrecciava con quello spagnolo. Ciò nel quadro del radicamento delle Scalze nel territorio italiano e della loro soggezione alla giurisdizione dell'Ordine o a quella dell'ordinario.

Anche in Sicilia, la cui provincia carmelitana fu inizialmente unita a quella di Roma e successivamente di Napoli, ${ }^{48}$ si assiste alla presenza di entrambe le tipologie, ed in particolare a Palermo sorsero ad un anno di distanza il monastero dell'Assunzione della B. V. Maria (1628), inizialmente di giurisdizione vescovile, e quello di S. Teresa (1629), dipendente dall'Ordine: entrambi fondati da due donne dell'alta aristocrazia; entrambi oggetto di «negozi»e

${ }^{46}$ Sulle alleanze matrimoniali intercorse fra Italia e Spagna e sulla politica di onorificenze elargite dalla corona ispanica agli aristocratici italiani fra Cinque e Seicento cfr. Spagnoletti A. 1996. Principi italiani e Spagna nell'età barocca, Milano: Bruno Mondadori.

${ }^{47}$ Contreras, J. 2009. «Procesos culturales hegemónicos: de religión y religiosidad en la España de Antiguo Régimen» Historia social 35: 3-22.

${ }^{48}$ Il capitolo generale del 15 maggio 1617 stabilì che la Sicilia e il Regno di Napoli fossero unite alla provincia romana; il 31 maggio 1620 la proposta di separazione dei due territori meridionali dalla provincia romana fu respinta. Fu accettata nel capitolo del 9 maggio 1626, che istituì la «Provincia Neapolitana, quae debet continere Regnum Neapolitanum utrasque Sicilias et insulam Melitam». Il 13 maggio 1632 «Propositum fuit an erigenda sit provincia Siciliae, quae debet continere Regnum Siciliae et insula Melitae, et dividenda a provincia neapolitana. Responsum est affirmativus cum 18 suffragiis», Monumenta Historica Carmeli Teresiani 11, Acta Capituli Generalis OCD Congregationis S. Eliae, vol. I, Antonio Forte (ed.) 1990: 66,116, 239, 288. Romae: Teresianum. Un panorama del Carmelo Scalzo siciliano in Gianninoto G. 1986. Mistero che attira. Per una storia del Carmelo teresiano in Sicilia, Villasmundo: Noviziato di Locomonico. Sul ramo feminile, cfr. il cap. III Le carmelitane scalze: 75-88.

Hispania Sacra, LXVII

136, julio-diciembre 2015, 467-503 ISSN: 0018-215X, doi: 10.3989/hs.2015.014 
dispute; entrambi divenuti oggetto di una mitologia delle origini, rielaborata da chi vi prese parte in prima persona o vi volle successivamente scorgere la manifestazione di una historia salutis che passava attraverso la celebrazione di una dinastia e dei suoi rapporti con le élites civili e religiose di Palermo, con i vertici dell'Ordine e con gli altri monasteri teresiani; soprattutto con quello napoletano di S. Giuseppe, da cui provennero le fondatrici dei due istituti cittadini.

All'incrocio di queste dinamiche si collocano le donne che trascorsero gran parte della loro esitenza nei «recinti» teresiani di quella che fu chiamata la felice città di Palermo: protagoniste e allo stesso tempo strumento di una più generale storia del potere, di cui i monasteri femminili costituiscono un segmento importante. Una storia che, sia nei suoi aspetti formali esterni che in quelli interni al governo delle singole istituzioni, «appare largamente condizionata dai modi e dai tempi di affermazione dello Stato moderno, ma al contempo è essa stessa elemento costitutivo, per minore che esso possa apparire, di quella dialettica». ${ }^{49}$

Sorti nella Palermo sede del viceré Francesco Fernandez de la Cueva, duca di Alburqueque, e dell'arcivescovo Giannettino Doria, membro della potente famiglia genovese, ${ }^{50}$ i monasteri dell'Assunzione e di S. Teresa vennero ad aggiungersi nei tardi anni Venti del Seicento alle fondazioni nate precedentemente in Italia. Sorretti dall'impegno e dal denaro dell'aristocrazia filo-spagnola, essi s'istallarono prima della canonizzazione della fondatrice spagnola, sull'onda della diffusione delle sue opere e della propaganda fatta dagli Scalzi nei territori in cui si erano già stabiliti.

È quanto appare confermato, ad esempio, dalle vicende di alcune teresiane di cui ci sono pervenute le vite esemplari: come quella della maltese Geltrude di Gesù Maria, la cui zia, entrata fra i quattordici e i quindici anni nell'antico monastero benedettino di S. Scolastica nella «città vittoriosa di Burgo», si trovò ad avere tra le mani una vita di Teresa d'Avila, «in Malta non ancora conosciuta non essendo stata ancora canonizzata [...] La lesse e letta ch'ebbe la vita con le fondazioni del S.mo suo Monasterio si accese talmente nel desiderio di

\footnotetext{
${ }^{49}$ Novi Chavarria, E. 2001. Monache e gentildonne, un labile confine. Poteri politici e identità religiose nei monasteri napoletani (secoli XVI-XVII): 21-22. Milano: Franco Angeli. Sui destini femminili - la monacazione o il matrimonio - nell'Italia postridentina cfr. Zarri, G. 2000. Recinti. Donne, clausura e matrimonio nella prima età moderna Bologna: Il Mulino.

${ }^{50} \mathrm{Sul}$ rapporto fra ptere politico e potere ecclesiastico, di cui i riti e le cerimonie profane e sacre erano espressione, Scalisi, L. 2004. Il controllo del sacro. Poteri e istituzioni concorrenti nella Palermo del Cinque e Seicento, Roma: Viella. Sul viceré si veda Di Blasi, G.E. 1791. Storia cronologica dei viceré, luogotenenti e presidenti del Regno, tomo II, parte II, 122-137 Palermo: dalle stampe di Solli. Su Giannettino Doria, arcivescovo di Palermo dal 1608 al 1642, Sanfilippo, M. 1992. Doria Giannettino, in Dizionario Biografico degli Italiani, vol. 41: 345-348. Roma: Istituto dell'Enciclopedia Italiana.
} 
imitarla che incominciò a proponere et a pregare ancora sua Madre che volesse con la sua sostanza e con le figlie fare un monastero simile a quello della S.ta Madre non havendo ancora notizia che vi fosse riforma e che la S.ta Madre passò innanzi non fermandosi in quelli unico monasterio, havendone fatti oltre a quello di molti altri di Donne e di Uomini et li nostri Padri non erano ancora in Malta». ${ }^{51}$

Ritorneremo più oltre sulla biografia di questa religiosa maltese monacatasi a Palermo, per la quale si ritenne opportuno raccogliere le sue vicende e i miracoli in vista di una beatificazione, di cui comunque non fu intrapreso l'iter. Se qui l'abbiamo richiamata è per anticipare un elemento che accomuna la maggior parte delle storie che hanno come scenario i monasteri della capitale del Viceregno: la presenza di forti figure femminili che, pur muovendosi all'interno di codici maschili e decisioni prese da padri, mariti, direttori spirituali, esibiscono un'intraprendenza e una capacità di gestire la propria vita e quella della istituzione di cui presero l'abito, modellate sull'esperienza di fondatrice di Teresa d'Avila. Una caratterisica, questa, che se da un canto rimanda al topos agiografico inevitabile in una documentazione destinata a celebrare $i$ fasti dell'Ordine, dall'altro ci fa riflettere su un modello di comportamento che verosimilmente attrasse le donne, soprattutto delle élites - o che fu da esse accettato - per la sua capacità di dare contemporaneamente voce alle istanze di perfezione religiosa provenienti dai movimenti di riforma cinquecenteschi e alle strategie patrimoniali e successorie delle ispanizzate aristocrazie italiane. ${ }^{52}$

Si legge ancora in una biografia dedicata ad un'altra carmelitana scalza nata a Palermo nel 1586 ma trasferitasi in Spagna per maritarsi - Luisa Moncada e Aragona, esponente di una casata che sarà protagonista delle prossime pagine che a undici anni si trovò fra le mani una vita manoscritta di santa Teresa «e ponendosi a carteggiarla con attenzione ben fissa, lesse ne' primi fogli, come la Santa Vergine ancora fanciulletta, dal leggere vani racconti di cavalieri erranti stette in procinto di traviare, e se bene in grave colpa non cadde, incespò nella vanità invaghita d'ornamenti». ${ }^{53}$ Le ferree strategie matrimoniali

\footnotetext{
${ }^{51}$ Brieve relazione della Vita, Morte e Miracoli della Madre Soro Geltrude di Gesù Maria, religiosa Scalza Carmelitana Professa nel Monasterio di S. Teresa della città di Palermo scritta per ordine del molto reverendo padre Fra' Eliseo di sant'Agnese, Provinciale del Medesimo ordine nella Provincia di Sicilia, ms. in AGCS, Pluteo 21, cartella M, f. 6v.

${ }^{52}$ Zarri, G. 1994. Dalla profezia alla disciplina (1450-1650), in Scaraffia L., Zarri G. (ed.), Donne e fede 177-225. Roma-Bari: Laterza. Per un esempio di confluenza fra istanze spirituali e strategie aristocratiche nella Sicilia del '600 cfr. Cabibbo, S. Modica, M. 1989, La santa dei Tomasi. Storia di suor Maria Crocifissa (1645-1699), Torino: Einaudi.

${ }^{53}$ Della Lengueglia A. 1652. Ritratti della prosapia et heroi Moncadi nella Sicilia, Valenza: per Vincenzo Sacco impressor viceregio, vol. II. Ritratto undecimo, Di donna Luisa Moncada, contessa di Santa Gadea e Buendia, poi monaca scalza carmelitana: 348. Una biografia della nobildonna si trova anche nel volume Riforma de' Scalzi di N.S. del Carmine dell'osservanza primitiva fatta da S. Teresa
} 
della famiglia le avrebbero consentito di vestire l'abito teresiano solo molti anni più tardi quando, dopo due infelici matrimoni e due vedovanze, sarebbe entrata nel 1625 nel monastero delle descalzas di Palencia, dove sarebbe morta quattr'anni dopo.

\section{DUE FAMIGLIE, DUE MONASTERI}

Nei tardi anni Venti del XVII secolo, consolidatasi la presenza a Palermo degli Scalzi giunti nel 1610, ${ }^{54}$ Antonio Moncada e Aragona, duca di Montalto e principe di Paternò, esponente di una delle più illustri famiglie siciliane la cui presenza percorre tutto il Medioevo e l'età moderna ${ }^{55}$ decise di fondare a Palermo un monastero di Scalze, forse ispirato anche dall'ingresso della sua congiunta - la già citata Luisa Moncada - nel monastero di Palencia. La decisione di impiantare nella capitale del Viceregno questa istituzione fu condivisa dalla moglie: Giovanna della Cerda, figlia del duca di Medinaceli, membro di una casata che aveva sostenuto le fondazioni teresiane in Spagna e parente di quella Luisa della La Cerda presso cui nel 1562 Teresa d'Avila aveva trovato ospitalità. Ottenuto il breve papale di separazione de thoro et mensa, la duchessa lasciò la famiglia e la città per monacarsi nel 1626 nel convento napoletano di S. Giuseppe a Pontecorvo, in cui prese il nome di suor Teresa dello Spirito Santo.

La documentazione conservata presso l'Archivio Generalizio dei Carmelitani Scalzi (d'ora in poi AGCD) fornisce informazioni sul «negozio» non limpido che precedette la fondazione palermitana del monastero dell'Assunzione, della cui progettazione fece probabilmente parte la precedente monacazione napoletana di Giovanna della Cerda: una scelta che, nell'intenzione della duchessa e del consorte, avrebbe favorito l'invio dalla città partenopea delle fondatrici dell'erigendo monastero siciliano. Si tratta di un'ipotesi che sembra confermata dalla decisione «segreta» del Definitorio Generale degli Scalzi del 26 agosto 1627 in cui si legge: «Assignatae sunt pro fundatione nn. Monasteri Panormi

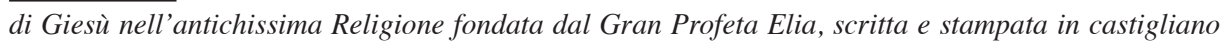
dal M.R.P.F. Giuseppe di Santa Teresa suo historico generale e tradutta nell'italiana da un Religioso del medesimo Ordine, 1701. Parma: per Alberto Pazzoni e Paolo Monti, tomo quarto, libro XIV, Vita dell'eccellentissima Signora Donna Luisa di Moncada, Aragona, contessa di S. Gadea nello stato di donzella e maritata: 602-610.

${ }^{54}$ Sull'insediamento degli scalzi a Palermo per iniziativa del viceré Juan Francisco Pacheco, marchese di Villena e duca di Escalona e del frate catalano Domenico di Gesù Maria, e sui due monasteri femminili, cfr. il panorama tracciato da Mazzarese Fardella, E. 2007. «Aspetti della religiosità in Sicilia nei secoli XVI-XVII. I Fardella-Paceco», Archivio Storico Siciliano, serie IV», XXXIII: 7-42.

${ }^{55}$ Sulla famiglia cfr. Laudani, S. 2008. Lo Stato del Principe: I Moncada e i loro territori, Palermo: Sciascia editore; Giarrizzo, G. 1999. «Alla corte dei Moncada», Annali di Storia moderna e contemporanea, 5: 429-436. 
m. Dorothea priorissa nunc Neapoli, sor Maria Hieronima et sor Teresia a Spiritu Sanctu cum obligatione definitorum ad secretum servandum circa nominatione». .6

Ma le cose non andarono come i coniugi avevano previsto, e soprattutto come il duca di Montalto premeva che si svolgessero, sollecitando Urbano VIII Barberini a concedere un breve che permettesse a sua moglie e ad altre due monache di uscire dal monastero napoletano, insieme ad una quarta religiosa: Maria Geronima del SS. Sacramento, «la quale era quella che teneva in sua cura la figliola della duchessa chiamata d. Anna Maria Moncada»: una presenza, quest'ultima, a cui la duchessa teneva molto perché la piccola era affezionatissima alla sua bambinaia velata, arrivando a chiamarla «mamma».

Ottenuta finalmente la licenza papale il 9 febbraio 1627 che «volendo potessero le monache uscire dal proprio monasterio per dar principio alla fondatione in Palermo $[\ldots]$ con facoltà che potessero tornare in quello di Napoli quando volessero», sorsero nuove difficoltà. Anzitutto quella di evitare il parere sfavorevole del Provinciale degli Scalzi, fra' Ferdinando di Santa Maria: un ostacolo che fu superato con il coinvolgimento dell'arcivescovo di Napoli, Francesco III Boncompagni. In secondo luogo intervenne la ferma opposizione di suor Geronima del SS. Sacramento a recarsi in un monastero che non fosse sottoposto alla giurisdizione dall'Ordine. E ciò nonostante le pressioni dell'arcivescovo napoletano e della duchessa di Montalto che «alla detta madre fece grandissime promesse et offerte $[\ldots]$ acciò la movesero a consolarla di andar con essa, ma lei sempre intrepida rispondendo in tutte l'occasioni: «Madre Teresa mia se sarà sotto la nostra Religione verrò volentieri, se sarà sotto l'ordinario mi teneria per scusata»» ${ }^{.57}$

La fonte di queste informazioni - una relazione anonima di un carmelitano scalzo - si dilunga sul furtivo allontanamento dal monastero napoletano della duchessa e delle sue compagne, che si diressero in carrozza verso la casa di una gentildonna napoletana. Ma la fuga, di cui erano forse a conoscenza i gesuiti, fu interrotta dal Provinciale che scongiurò il pericolo che le tre religiose alloggiassero «in case di secolari et in tempo di carnevale». Rientrate come ospiti presso il S. Giuseppe, esse subirono così l'onta di vivere per qualche tempo fra le mura di quel monastero dove avevano ricoperto delle cariche; in seguito, in attesa della partenza per Palermo, esse trovarono accoglienza in una comunità di francescane..$^{58}$

\footnotetext{
${ }^{56}$ Forte A. (ed.) 1985. Monumenta Historica Carmeli Teresianim Subsidia 3, Acta Definitorii Generalis OCD Congregationis S. Eliae, Libro I, Regesta Actorum 1617-1627, 146. Romae: Teresianum.

${ }^{57}$ Relazione dell'uscita della Madre Theresa del Spirito santo dal Mon.ro di san Gioseppe di Napoli e della sua venuta qui a Palermo, AGCD, Sezione A, Pluteo 121, Cartella G, documento 3.

${ }^{58}$ Ivi. Il cronista si sofferma sulla consegna delle chiavi del monastero da parte della madre Dorotea [Mercante], che aveva ricoperto la carica di priora.
} 
Anche la lettera inviata alla vicaria e alle religiose napoletane dal Provinciale al momento del provvisorio rientro delle tre fuggitive nel monastero di san Giuseppe fa riferimento a questi avvenimenti che dettero «uno scandalo grande». Cercando di orientare il comportamento della comunità, egli suggeriva: «V.V.R.R. tengano carità con loro che sono sorelle sue et figlie mie, no si senta parlar de puntura né disgusto, ogni una atenda a se et de opere e parole le tratino benisssimo»..$^{59}$

Sbarcate infine nel porto palermitano il 20 giugno del 1628, la duchessa di Montalto insieme alla figlioletta e alle due consorelle si diressero al monastero dell'Assunzione della Beata Vergine Maria, di giurisdizione vescovile, situato in un palazzo di Via Maqueda, già dotato di una chiesa fatta costruire dal duca ${ }^{60}$ Magnifica - raccontano le cronache - fu la cerimonia, a cui parteciparono il viceré, l'arcivescovo e tutta la nobiltà palermitana, rendendo omaggio ad un Ordine che impiantava il suo ramo femminile nella capitale del Viceregno e a quella famiglia di «heroi Moncadi», giunta nel XIII secolo nella Sicilia aragonese e qui divenuta sempre più potente sino a vantare tra le sue fila un viceré, Ugo Moncada; una casata le cui origini affondavano, secondo la fantasia genealogica dell'epoca, nella popolazione germanica dei Bavari stanziatatisi nella penisola iberica. ${ }^{61}$

Qualche tempo dopo anche il duca di Montalto, affidati al figlio titolo e feudi, abbandonò il secolo ed entrò nella Compagnia di Gesù presso cui sarebbe morto nel 1631. L'evento fu seguito dalla richiesta delle religiose dell'Assunzione di passare sotto la giurisdizione dell'Ordine e dalla relativa bolla papale del 14 aprile 1632, registrata dal definitorio generale degli Scalzi. ${ }^{62}$ Sotto il lungo priorato di Madre Dorotea il monastero prosperò, accogliendo le vocazioni di altre aristocratiche e proponendosi anche come rifugio per nobildonne secolari, fino a diventare nel corso del secolo il «recinto» di molte donne della famiglia

${ }^{59}$ Lettera del N.P. Ferdinando alle Monache di Napoli per il modo improprio d'uscire le fondatrici di Palermo, AGCS, Sezione A, Pluteo 121, Cartella G, documento 2.

${ }^{60} \mathrm{Sul}$ monastero cfr. anche A. Mongitore, Storia sagra di tutte le chiese, conventi, monasteri, ospedali e altri luoghi pii della città di Palermo, Monasteri e conservatori, ms. XVIII sec., Biblioteca Comunale di Palermo, Qq E 7: 123 ss. I testi riferiscono che il duca, acquistato il terreno e edificato il monastero, assegnò a quest'ultimo un capitale fisso e una dote, arricchendo la chiesa di reliquiari e altre preziose suppellettili, per una cifra complessiva di circa 100.000 scudi.

${ }^{61}$ Su Ugo Moncada, viceré di Sicilia (1509-1516) e di Napoli (1527-’28) e sul suo rapporto con i sovrani spagnoli, Bazzano N. 2011. Moncada, Ugo, in Dizionario Biografico degli Italiani, vol. 75: 578-583. Roma: Istituto dell'Enciclopedia Italiana. Sulla genealogia della casata, Della Lengueglia, A., I «ritratti della prosapia... cit.; sull'opera e il suo autore, Cabibbo, S. 1995. «Un'opera storica encomiastica nella sicilia del Seicento: I «ritratti della prrosapia et heroi Moncadi» di Agostino della Lengueglia», in Benigno F. e Torrisi C. (eds.), Città e feudo nella Sicilia moderna: 137-152. Caltanissetta: Salvatore Sciascia editore.

${ }^{62}$ Acta Definitorii Generalis, cit., vol. I: 203. 
Moncada. Come quando fu approvata la richiesta di far entrare nel monastero di Napoli la tredicenne Giovanna, figlia di Ignazio Moncada e Aragona e nipote dei fondatori, e di accogliere a Palermo «ad educationem» la sorella undicenne Antonia. ${ }^{63}$ Quanto alla duchessa, suor Teresa dello Spirito Santo, se ne conoscono i successivi spostamenti presso la comunità napoletana e in Spagna, dove le fu consentito di recarsi per dare vita ad una nuova fondazione. ${ }^{64}$

Un percorso di fondazione più lineare fu quello del monastero di S. Teresa, di cui si trova per la prima volta notizia il 9 marzo del 1627, allorché il definitorio generale degli Scalzi ne approvò l'istituzione, precisando che era necessaria la presenza di almeno tre fondatrici. Anch'esso fu legato ad una potente famiglia - quella dei Fardella - che pur non potendo esibire la prestigiose origini feudali dei Moncada e il costante potere da essi esercitato nei secoli, riuscì fra Cinque e Seicento a conquistarsi un posto di primo piano nel panorama aristocratico siciliano, grazie ad un'accorta politica matrimoniale e alla fedeltà dimostrata alla corona spagnola. In un breve spazio di tempo, infatti, questa laboriosa famiglia della piccola nobiltà trapanese, precedentemente composta da funzionari ed amministratori locali arricchitisi con il possesso di saline e tonnare, celebrò nel 1607 il matrimonio di un suo esponente, Placido Fardella di soli quindici anni, con la nipote del nuovo viceré giunto nel'isola un anno prima, Anna Maria Pacheco y Mendoza: un'unione che procurò allo sposo l'investitura del feudo di Paceco e la licenzia aedificandi et populandi una città di cui fu nominato principe, rappresentando un perfetto esempio di mobilità sociale in una Sicilia in cui gli Austrias dettero vita a quella che è stata definita «rifeudalizzazione ${ }^{65}$

${ }^{63}$ Ibidem, 12 agosto 1651: 481.

${ }^{64}$ Il 26 marzo 1635 «Conceditur ut M. Teresa de Spiritu Santo, priorissa mon. Assumptionis Panormi, simul cum Antonia Gertrude eius filia, transire possit ad Congreg. Hispaniae ut ibi faciat fondationem monialium»; il 23 ottobre 1636 «Datur licentia M. Teresa a Spiritu Sancto ut cum m. Dorotea a S. Anna transire possit a monasterio Assumptione Panormi ad monasterium S. Joseph Neapolis; ea tantum conditione quod Ex.mus Princeps de Paternò, filius eiusdem $\mathrm{m}$. Teresiae, assignaverit nomine mon. Panormi annuos reditus et quidquid reliquit pro dotatione praedicti monasterii» (Acta Definitorii Generalis, cit.: 264 e 275).

${ }^{65}$ Sulla famiglia Fardella cfr. Nocella P. L. 2010. Lealtad a la corona y ascenso social de una familla siciliana, in Familias, jerarquización y movilidad social, in Levi G., Rodríguez Pérez R. (eds.), 341352. Murcia: Universidad de Murcia. Sulla concessione da parte dei sovrani spagnoli di titoli e territori a uomini siciliani e sul conseguente fenomeno di ascesa sociale da essi compiuto perché il possesso di un feudo popolato garantiva l'ingresso nel braccio militare del parlamento dell'isola e l'attribuzione di un titolo feudale, Ligresti, D. 2010. Centri di potere urbano e monarchia ispanica nella Sicilia del $X V$-XVIII secolo, in Centros de poder italianos y monarquía hispanica (siglos XV-XVIII), J. Martinez Millan y M. Rivero Rodríguez (eds.), vol. III: 287-329. Madrid: Ediciones Polifemo; Pinzarrone L. 2014. «La politica delle fondazioni feudali nella Sicilia del XVII secolo: procedure, controversie, giurisdizioni», Storia urbana, 142: 5-20.

Hispania Sacra, LXVII

136, julio-diciembre 2015, 467-503 ISSN: 0018-215X, doi: 10.3989/hs.2015.014 
La principessa Anna Maria Paceco, vedova del giovane principe morto di peste nel 1623 e tutrice dei figli, sta alle origini di questo secondo monastero sulla cui ideazione le fonti divergono, mostrando un'opacità che rimanda anche qui ai rapporti fra il potere vescovile e l'Ordine degli Scalzi. Se infatti nella biografia di Maria Maddalena di S. Agostino - figlia della principessa e religiosa morta in odore di santità $-{ }^{66} \mathrm{i}$ carmelitani appaiono come gli autori della proposta avanzata alla principessa di Paceco di fondare il secondo monastero palermitano, in un altro documento l'iniziativa è presa dall'aristocratica, ben a conoscenza della spiritualità e delle pratiche religiose delle teresiane.

Nel primo dei due testi si legge infatti che la decisione del duca di Montalto di porre il monastero da lui fondato sotto la giurisdizione dell'ordinario era stata per il preposito generale: «un colpo [...] sentito con qualche amarezza», sicché l'Ordine «deliberò fondare un altro Monastero nel medesimo Palermo soggetto alla sua obbedienza. Per questo intento scrisse alla Principessa D. Anna Maria Paceco, rappresentandole il suo desiderio, e insieme offerendole il titolo di Fondatrice». La proposta fu accettata «di buon grado» dalla nobildonna che aggiunse di avere in animo di entrare essa stessa nell'istituto, una volta che «havessi collocati sì il Prencipe suo Figlio, sì Donna Cecilia, sua Figlia»: gli unici membri della casata per i quali non fosse stato già deciso il destino. Prosegue la fonte:

Con sì buon fondamento, e appoggio incominciossi da' nostri Superiori in Roma, e de' Religiosi a Palermo a trattare della nuova fondazione, e s'incontrarono subito gravi difficoltà $[. .$.$] Era la più ardua il negarsi dal Cardinale Giannettino d'Oria Arcivescovo$ di Palermo il suo consentimento: atteso che senza questo giammai sarebbesi in Roma spedito il Breve. A vincerla vi si adoperò con tutto l'ardore dell' animo suo la Principessa, e doppo molte suppliche, cedendo il Cardinale all'autorevole mediazione di sì qualificata Signora, e via più alle disposizione di Sua Divina Maestà, le scrisse un viglietto in lingua Spagnuola di questa sostanza. Sapere Sua Signoria Illustrissima, quanto fosse propenso a concorrere in quello, fosse di sua consolazione, e dipendesse dalla propria volontà, che però nell'istanza, da lei fattagli, non si farebbe da se opposizione, quando da Sua Santità s'impetrasse il necessario beneplacito. ${ }^{67}$

Se nel testo di Biagio della Purificazione la principessa di Paceco appare come la destinataria di un invito rivoltole dai padri dall'Ordine e l'autorevole intermediaria fra questi e l'arcivescovo palermitano, in un volume manoscritto che contiene una serie di relazioni di autori diversi e alcune biografie di

\footnotetext{
${ }^{66}$ Biagio della Purificazione 1703. Vita dell'insigne Serva di Dio la Madre Maria Maddalena di S. Agostino, carmelitana Scalza nel Monastero di S. Anna e S. Teresa a Palermo, Roma: Stamperia e Gettaria del Zenobi. L'opera era dedicata dallo storico dei carmelitani scalzi, autore di molte biografie di monache dell'Ordine morte in odore di santità, ad una nipote per via di padre della protagonista della biografia: Maria Fardella e Gaetani, principessa di Bisignano.

${ }^{67}$ Ibidem, 28-29.
} 
monache, la nobildonna appare la vera e propria fondatrice del monastero di S. Teresa, secondo quanto recita la «breve relación de heste monasterio hallada en los libros manuscritos pertinecientes al mismo escrita dal mui Reverendo Padre Fray Martial de Santa Maria carmelita descalzo y prior que fue del Conbento de S. Maria de los Remedios de los carmelitas descalzos de Palermo». ${ }^{68}$

Qui è la principessa di Paceco che chiede all'arcivescovo «de fundar un monasterio de monjas carmelitas descalzas con el nombre de S. Teresa debaxo el cuidado y govierno de los padres carmelitas descalzos, libres de la jurisdición de su Eminenza y sucesores, ma no pudo ser tan secreta esta domanda que no viniese a las orjas de muchas Personas poderosas entre las quales fueron algunas Religiosas de otra Religión que se movieron en un tiempo para empedir esta Sancta obra en que [...] muchas contraríedades y como eran movidas de Personas poderosas y de Dominio se tenía algun impedimento». ${ }^{69}$

Con grande prudenza lo scrivente non riportava i nomi delle personas poderosas che avevano dissuaso l'ordinario palermitano a porre l'erigendo monastero sotto la giurisdizione degli Scalzi; ma questa reticenza e la contraddizione fra le due fonti riflette probabilmente le tensioni che si coagularono anche intorno alla comunità di S. Teresa, che ebbe come prima sede un edificio molto modesto «en la subida de los Pozillos a lado de la muralla de la ciudad y en medio de la puerta de Mazara y aquella de santa Agada y allà se fabricò y pusò en forma de Monasterio con esperanza que Dios lo agradezesa y haza maior magnifico y celebre con la observancia y la santidad de sus siervas». Come fondatrici furono chiamate tre monache del monastero napoletano di S. Giuseppe: suor Teresa di S. Francesco e suor Maria Geronima del Santissimo Sacramento «ambas señoras napolitanas», e suor Maria Francesca di S. Ines «una de las señoras principales de Genova». ${ }^{70}$ Lasciato il loro comodo monastero per stabilirsi in uno più angusto e disagiato, le religiose si imbarcarono in una galera siciliana il 7 agosto 1629 e raggiunsero Palermo dopo sei giorni di navigazione.

\footnotetext{
${ }^{68}$ Questo testo costituisce il primo documento di un libro manoscritto che contiene inoltre due relazioni sulla fondazione e la traslazione dell'istituto scritte da Maria Maddalena di Sant'Agostino, protagonista della biografia di Biagio della Purificazione, molti documenti e lettere, tra cui quelle intercorse fra Anna Maria Paceco e l'Arcivescovo Doria, e undici biografie di monache. Il volume, «en el cual se cuentan las cosas mas memorables de aqueste monasterio de Santa Teresa», e che oggi è conservato conservato presso l'AGCD (Pluteo 421 a), era dedicato a Maria Sanseverino Fardella Pacheco y Caetano, principessa de Bisignano, di cui la fondatrice era «muy querida tia y por esta razon se debe tambien a V.R. que la ama de corazon». Del monastero fondato nel 1629 e del successivo in cui le monache si trasferirono, l'autore della dedica - Ferdinando Griso - scrive che furono costruiti con le «limosnas de su casa que son mas veinte y quatros mil excudos».

${ }^{69}$ Breve relacion de la fundacion de este monasterio hallada en los libros manuscritos, cit.

${ }^{70}$ Ibidem.
} 


\section{TERESIANE FRA MEMORIA E AGIOGRAFIA}

Il monastero di S. Teresa, che successivamente aggiunse nella sua titolazione il primo nome - Anna - della principessa di Paceco in ricordo della funzione da lei svolta nella fondazione, è rappresentato dalla documentazione esistente come un luogo di perfezione, dove si svolsero le vite edificanti di molte Scalze, degne, nella ricostruzione dei agiografi, di salire agli onori degli altari. In questo senso, la silloge manoscritta sopra citata costituisce probabilmente anche il tentativo di dare una cornice adeguata alle biografie di due figure, assai diverse fra loro per estrazione sociale, ma che riflettono entrambe alcune peculiarità del modello teresiano per le vicende di cui furono protagoniste prima della monacazione e per l'intraprendenza mostrata nello scegliere la regola teresiana. Si tratta di Cecilia Fardella, figlia della principessa di Paceco, monacatasi col nome di Maddalena di Sant'Agostino (1614-1690), e della già citata maltese Geltrude di Gesù Maria, nel secolo Timotea Cumbo (1613-1656). Gli scritti della prima, la cui biografia fu redatta da Biagio della Purificazione, furono raccolti dalle monache e inviati al carmelitano Giovanni Paolo dell'Epifania, e poi pubblicati a Venezia; ${ }^{71}$ della seconda esiste il manoscritto Brieve relazione della Vita, Morte e Miracoli della Madre Soro Geltruda di Gesù Maria, Religiosa Scalza Carmelitana professa nel monastero di S. Teresa della città di Palermo, scritta per ordine del molto Reverendo Padre fra' Eliseo di sant'Agnese, Provinciale del medesimo Ordine nella Provincia di Sicilia, composta dallo scalzo fra' Angelo Maria. ${ }^{72}$

La biogafia che Biagio della Purificazione dedica a Maria Maddalena di Sant'Agostino, in gran parte ricavata da una relazione di spirito composta dalla stessa carmelitana, si sofferma a lungo sul periodo che precede il manifestarsi della vocazione e la decisione di Cecilia Fardella di prendere il velo nel monastero fondato dalla madre. Educata secondo «il costume della Nazione Spagnuola più frequentato» per cui le le fu assegnata «al quarto anno un'Aja [...] di lodevolissimi costumi», scampata alla peste che uccise il padre e circa trenta persone

\footnotetext{
${ }^{71}$ La notizia è data da fra' Ferdinando Griso nella dedica del volume manoscritto, più sopra citato, a Maria Sanseverino Fardella e Paceco. Lo scritto di Maria Maddalena di Sant'Agostino, pubblicato a Venezia nel 1672 nella stamperia del Turrini, è la Fondatione e traslazione del monastero di Santa Teresa, delle carmelitane scalze, nella felice città di Palermo [...] ed elogi di nove religiose morte sin a questo tempo in detto monastero. Del testo ci fu una seconda edizione veneziana nel 1673. Quanto a Giovanni Paolo dell'Epifania, fautore della pubblicazione, si tratta del palermitano Antonio Parisi (1633-1676), entrato nell'Ordine degli scalzi nel 1653, docente di retorica, filosofia e teologia a Palermo e Messina, autore di molti scritti devozionali e di un'orazione funebre per la morte di Filippo IV. (Collectio scriptorum Ordinis Carmelitarum excalceatorum utriusque congregationis et sexus P. F. Bartholomei a S. Angelo Provinciae Longobardicae opera et solertia exarata, vol. I: 336-338. Savona: Typographia Ricci.

${ }^{72}$ Il manoscritto è conservato presso 1'AGCD, Pluteo 21, cartella M.
} 
dell'entourage familiare, destinata al matrimonio, la giovane aristocratica raggiunse l'adolescenza «crescendo il lei, con il conoscimento delle sue raguardevoli doti, l'affezione alle gale, e pompe secolaresche», compiacendosi di essere considerata «una delle più vistose donzelle della sua Patria». Inoltre, era solita leggere «libri di cavalleria, comunemente detti Romanzi; sapendo, che quell'ameno favellare servirebbe a rifiorire i suoi ragionamenti, e si addentrerebbe la sua lingua à detti di graziose piacevolezze». E tuttavia, aggiunge il biografo, «camminando per queste vie, non erasi peranco traviata [...] Confessò peraltro lei medesima, non havere fra tutti i predetti divertimenti oltre passato i limiti dell'onesto, e che nell'adornarsi, e compiacersi della sua naturale bellezza, non erasi giammai profanato il cuore con rea intenzione». ${ }^{73}$

Il tema teresiano dell'affezione al secolo e ai suoi piacevoli rituali costituisce il fulcro della prima parte della biografia, dedicata al periodo anteriore alla monacazione. Spostandosi tra Trapani, Paceco e Palermo al seguito degli impegni di una famiglia dell'alta aristocrazia, la giovane Cecilia alternava momenti di allegrezza e divertimento ad altri più riflessivi e travagliati; momenti in cui un'immagine sacra, l'invito del suo confessore gesuita «a fare di se medesima a Dio un gradito sacrificio», un sogno difficile da decifrare, o la particolare «intelligenza» che le dette il Signore di adattare a se stessa l'invito dell'Audi filia di abbandonare il secolo, la costringevano all'introspezione e a scegliere fra le due opzioni riservate alle donne: aut maritum, aut murum.

«Un accidente molto di rilievo» favorì la decisione di Cecilia, che aveva raggiunto i vent'anni.

«Era di quel tempo Viceré della Sicilia il duca di Albuquerche, e volendo il Re Cattolico sostituirgli in quel governo il Duca di Alcalà, le lettere venute dalla Spagna, gli portarono avviso della Regia deliberazione. Con questo dispaccio venne la lettera, scritta dal Marchese di Vigliena, quale era stato Viceré di questo Regno, alla Principessa di Paceco, e in essa eragli rammentato la promessa fattagli otto anni prima, di mandare nella Spagna Donna Caterina e Donna Serafina sue figlie a Monacarsi, come ne havevano mostrato desiderio, nel Monastero celebre di Madrid, detto il reale delle Francescane Scalze. Havere Sua Eccellenza ricevuto l'honore del rivevimento delle due mentovate sue Figlie: Volere pertanto tutte le convenienze, che più non differisse l'adempimento della sua parola [...] Riflettendo la Principessa al tenore di questa lettera, deliberò andar lei medesima in Spagna, e condurre seco per il predetto Monastero Donna Serafina solamente; atteso che Donna Caterina dimostravasi poco incline a monacarsi; e se l'havesse condotta, prevedeva l'esporrebbe a pericolo d'indurla

\footnotetext{
${ }^{73}$ Biagio della Purificazione, Vita dell'insigne Serva di Dio la Madre Maria Maddalena di S. Agostino, cit.: 16-17.

Hispania Sacra, LXVII

136, julio-diciembre 2015, 467-503 ISSN: 0018-215X, doi: 10.3989/hs.2015.014
} 
per humano rispetto allo Stato Religioso. Non voleva però lasciarla in casa secolare, ma nel nostro Monastero di S. Teresa, puotendosi entrare in educazione, per la dispensa concedutale dal Papa». ${ }^{74}$

La lunga citazione, che illustra un segmento della vita della futura «monaca santa», merita un commento rivolto a mettere in evidenza il ruolo che giocavano i monasteri femminili non soltanto nelle strategie delle famiglie aristocratiche - elemento messo in rilievo dalla storiografia,$-{ }^{75}$ ma anche nelle relazioni che intercorrevano fra la corte spagnola e i domini italiani degli Austrias. Lungi da configurarsi come semplici rapporti fra centro e periferia, fra dominatori e dominati, questi legami fra la monarchia e i ceti dirigenti dell'isola includevano, oltre alle alleanze matrimoniali che spingevano donne e uomini dei due paesi a trasferirsi nella terra del coniuge, anche gli spostamenti di religiose da uno Stato all'altro, sulla base di strategie e convenienze elaborate in anni che precedevano di gran lunga l'effettiva dislocazione. Come i conventi maschili, anche i monasteri femminili si configuravano quindi come luoghi di socializzazione, di «internazionalizzazione», di scambio e costruzione di relazioni, utili a promuovere il processo di ispanizzazione dei ceti dirigenti.

Dimostrata una particolare sensibilità per la dubbia vocazione della figlia Caterina, inviata al monastero di famiglia «per educazione», ${ }^{76}$ la principessa Anna Maria Paceco partì dunque alla volta di Madrid con la figlia Serafina, dopo aver stabilito che Cecilia andasse ad abitare a casa di una parente, senza aver sospetto che ella incominciava «quasi a cadere agl'impulsi della Divina Grazia». Ciò, scrive il biografo basandosi sulla relazione di spirito da essa scritta dopo la monacazione, determinava l'alternarsi di fugaci allegrie per gli svaghi procurati dalla famiglia ospitante e di penosi pensieri che sorgevano dopo aver meditato a lungo davanti ad un'immagine dell'Hecce homo: un'alternarsi di sentimenti e dubbi a cui non davano requie i colloqui col confessore del tempo, il gesuita Mariano Prasis, «che non era favorevole alla vocazione della sua figlia spirituale».

La decisione di entrare in monastero, dissimulando con il fratello - il giovane principe di Paceco - e con gli altri parenti di voler andare a trovare la sorella

\footnotetext{
${ }^{74}$ Ibidem, 36-37.

${ }^{75}$ Sul carattere aristocratico dei monasteri femminili del XVII secolo e sull'esistenza di «monasteri di famiglia» in cui si monacavano le donne della dinastia, giungendo a trasmettersi le celle, cfr. Medioli, F. 1997. La clausura delle monache nell'amministrazione della Congregazione romana sopra i Regolari, in Zarri, G. (ed.), Il monachesimo femminile in Italia dall'altro medioevo al secolo XVIr: 249-282. Verona: Il segno dei Gabrielli.

${ }^{76}$ Il biografo sottolinea che la principessa di Paceco chiese all'arcivescovo di Palermo di inviare nel monastero un suo vicario «affinché esplorasse la volontà di Donna Caterina, non volendo che rimanesse nel nostro Monastero, se non vi concorresse la sua propria» (Biagio della Purificazione, Vita dell'insigne Serva di Dio la Madre Maria Maddalena di S. Agostino, cit: 38).
} 
educanda presso il S. Teresa, fu presa all'improvviso, all'età di ventun'anni. Non fermandosi neanche a salutare tutti coloro che avevano rappresentato fino ad allora i suoi affetti più cari, Cecilia montò in carrozza «e se alla nostra Madre Teresa quando partissi dalla Casa del Padre per andare al Monastero pareva se le slogassero per la pena le giunture del corpo, a questa futura sua figlia sembrava le fosse da morsi de' leoni sbranato il cuore». ${ }^{77}$

L'impronta del modello teresiano, così spiccata nei primi capitoli della biografia, e ribadita dal nome che Cecilia volle darsi in religione - Maria Maddalena di S. Agostino, a metafora del conflitto fra l'amore terreno e amor di Dio che aveva travagliato la sua giovinezza - impronta anche il resto del testo, in cui le «prudenti maniere» con cui esercitò per sei volte la funzione di priora, la cura che dedicò alle consorelle nel guidarle nel cammino di perfezione religiosa, inducono il biografo a ribadire il debito che ella contrasse con la fondatrice spagnola.

«Le priore hanno a por mente, che non sono state poste in quel luogo perché a loro piacere eleggano il cammino, e secondo i dettami del proprio spirito, ma perché conducano le suddite per quello le viene prescritto dalla loro Regola, e Costituzioni, ancorché si facciano violenza per essere inclinate ad altri dettami», postilla l'agiografo, aggiungendo che la prudente priora, per rispettare questa indicazione, rileggeva continuamente questi testi e «ponderando alcuni punti, scrisse intorno a' medesimi alcune profittevoli riflessioni appoggiandole alla dottrina dei Santi Padri, palesando non solo la vivacità del suo ingegno, ma l'elevatezza del suo Spirito nel penetrare i più veri sentimenti delle sue santissime leggi». ${ }^{78}$

Lungo i tre libri in cui si articola la biografia, Biagio della Purificazione ripercorre la vita edificante della figlia dei principi di Paceco, i suoi travagli umani e spirituali, le virtù di cui dette prova, i patimenti e le sofferenze che l'afflissero sin dai giorni antecedenti la sua solenne professione ${ }^{79}$ fino alla

${ }^{77}$ Ibidem: 50.

${ }^{78}$ Ibidem, 131. Non ho preso visione degli scritti sulle Costituzioni che, secondo Biagio della Purificazione erano conservati presso il monastero di S. Teresa. A proposito di questa attività di suor Maria Maddalena di S. Agostino, egli scrive: «Né in ciò contravvenne all’Apostolo, qual prescrisse alle Donne 1'imparar tacendo [...] essendo nota l'interpretazione dell'Angelico Tomaso, che afferma ciò doversi intendere nella pubblica predicazione del Vangelo: e però San Paolo intimando la predetta prohibizione saviamente soggiunse, che non insegnino nella Chiesa, qual è l'adunanza dei fedeli» (315). Sulla figura della priora cfr. Janke-Leutzsch, G.1992. Clara Staiger, la priora, in Calvi G. (ed), Barocco al femminile: 97-126, Roma-Bari, Laterza.

${ }^{79} \mathrm{Il}$ biografo precisa che, per la rinuncia di Cecilia ai beni che le erano stati assegnati in previsione della sua permanenza nel secolo e del matrimonio, il fratello principe pagò 5000 scudi in contante per la fabbrica del monastero, impegnandosi a versare 100 scudi ogni anno e 100 scudi di rendita a ragione del 5\% (Ibidem: 109).

Hispania Sacra, LXVII

136, julio-diciembre 2015, 467-503 ISSN: 0018-215X, doi: 10.3989/hs.2015.014 
morte per «mal di pietra» avvenuta il 7 novembre 1694, a ottant'anni di età, di cui cinquantuno trascorsi in monastero. Di questa vita, fin qui narrataci dall'agiografo, vogliamo cercare di recuperare un frammento della voce di Maria Maddalena di S. Agostino, affidata alla relazione che ella compose, in terza persona e in spagnolo, sulla Traslacion del Monasterio de Santa Teresa.$^{80}$ In queste pagine, consultate nella copia manoscritta, la carmelitana scalza si autorappresenta come vera figlia ed erede di Teresa d'Avila, soprattutto quella delle Fondazioni, soffermandosi puntualmente sulle difficoltà e sugli aiuti celesti e umani che accompagnarono il trasferimento delle moniali da un lugar giudicato dal principe di Paceco «poco combeniente para un monasterio de tanta autoridad y exemplar como por gracia de Dios son en todas las Ciudades» ${ }^{81}$ ad un altro che rispecchiasse il prestigio dell'Ordine e della famiglia fondatrice.

Il particolareggiato resoconto delle trattative avviate e non portate a termine per ottenere un terreno o un edificio da adibire a nuovo monastero, la puntigliosa citazione delle persone d'alto rango coinvolte in questi negocios, lo scarto fra la fiduciosa attesa delle monache di trovare il sitio da esse desiderato e il disincanto dei padri carmelitani che giudicavano fantasias de las mujeres le aspirazioni delle religiose: tutto ciò richiama le Fondazioni teresiane, come se l'autrice della relazione, non potendo percorrere in lungo e in largo la Spagna alla ricerca di seguaci e benefattori che patrocinassero i nuovi istituti, percorresse le opportunità offerte dalla città e dai suoi aristocratici abitanti per ottenere il sospirato, nuovo monastero.

Sfumate una serie di possibilità che si aprivano nella Palermo alle prese con una febbre immobiliare che coinvolgeva individui e istituzioni civili e religiose, facendo aumentare i prezzi da un giorno all'altro ${ }^{82}$ - come nel caso di un edificio individuato dalla famiglia Paceco, ma acquistato dalla principessa di Rocca Fiorita «para la fundacion de un monasterio de la Madre Sor Ursula y tan antes havia pasado dicho concierto que ya era hecho el acuerdo y dicha Princesa ofrecía diez mil ducados por prezio de dicha casa» - suor Maria Maddalena di sant'Agostino e le sue consorelle affontarono con serenità e pertinacia tutte le avversità che di volta in volta allontanavano la speranza di installarsi in un nuovo sitio: la minaccia turca e la guerra di Candia, che impegnarono le autorità rendendole poco sensibili alle esigenze delle monache, l'affollamento del

\footnotetext{
${ }^{80}$ Traslacion del Monasterio de Santa Teresa escrita de la Madre Maria Magdalena de San Augustin Carmelita Descalza, AGCD, Pluteo 421 A. Del testo esiste un'edizione a stampa, Fondazione e traslazione del Monastero di Santa Teresa [...] scritte dalla Madre Maria Maddalena di S. Agostino [...] ed elogi di Nove Religiose morte sino a questo tempo in detto Monastero, cavati dagli scritti del Rev. P. F. Gio. Paulo dell'Epifania, Carm. S. cronista della provincia di Sicilia, 1672. Venezia: Turrini.

${ }^{81}$ Traslacion del Monasterio, cit.: 17.

${ }^{82}$ Di Matteo. 2002. Palermo, storia della città dalle origini ad oggi: 93-97. Palermo: Kalos.
} 
vecchio edificio in cui entrarono molte nobildonne, l'ostilità di altri monasteri, poco propensi ad avere come vicine le teresiane. Avvenimenti tutti che la scrivente descrisse minuziosamente accompagnadoli con la menzione degli scudi impegnati di volta in volta per il nuovo monastero, finalmente «conquistato» nel 1653. Terminava così un'epopea che per l'autrice della relazione «comenzo come burlando entre nosotros y muy maiormente entre nuestros Padres que ercieron al fin que lo tratado no haria de tener efecto en pensamiento y fantasias de las mujeres». ${ }^{83}$ Anche in questa notazione, che rimanda al rapporto fra religiosi di diverso sesso si rispecchia lo spirito di Teresa d'Avila e la sua «retorica della femminilità». ${ }^{84}$

Se lo scenario che fa da sfondo alle vicende di Maria Maddalena di S.Agostino è la Sicilia dei viceré e dell'aristocrazia, con la sua capitale che si configura come un distaccamento della corte madrilena, le origini di Timotea Cumbo, entrata nel monastero di $\mathrm{S}$. Teresa nel $1632 \mathrm{col}$ nome di suor Geltrude di Gesù e Maria, affondano nell'isola di Malta, in cui la famiglia viveva da più generazioni. In mancanza di nobili natali, il biografo dedicò ampio spazio ai pii costumi e all'onorevole posizione sociale dei suoi antenati, alcuni dei quali dottori in legge e familiari dei cavalieri di Malta, e alla devozione delle sue donne, fra cui suor Maria della Croce «monaca scalza del nostro Monastero dell'Assunta nella città di Palermo fondato dall'Ill.mo Signor D. Antonio Moncada, duca di Montalto». 85

Composto per avviare l'iter di beatificazione, come dimostra il titolo della relazione che include anche i miracoli della carmelitana, il testo del confratello Angelo Maria avvolge in un'atmosfera miracolosa tutta l'esistenza della protagonista, a partire dai mesi precedenti la nascita. Alla madre che di lì a poco sarebbe rimasta vedova del secondo marito, infatti, apparve in sogno una bellissima giovane donna che, smontata da una carrozza, «pigliando con le mani il Corpo di lei già gravida di Geltruda, le diceva con molta grazia in questa guisa: Mi vuoi tu dare questa Rosa per la quale son venuta? E rispondendo Giovanna prontamente: Signora, quanto vi è è tutto vostro, la Signora rimontò in carrozza». ${ }^{86}$

Gli eventi eccezionali si susseguono in questo testo, fortemente impregnato di quell'agiografia leggendaria che, a dispetto degli appelli dell'erudizione ecclesiastica, continuava a godere di molto favore presso i monasteri femminili,

\footnotetext{
${ }^{83}$ Ivi. Presso l'AGCD si trova traccia delle richieste avanzate dalle scalze di S. Teresa per ottenere il permesso di fondare un nuovo monastero (Acta Def. Gen., vol. 3, 441, 475, 613).

${ }^{84}$ Weber, A. 1993. Teresa d'Avila e la retorica della femminilità, Firenze: Le Lettere.

${ }^{85}$ Brieve relazione della Vita Morte e Miracoli della Madre Soro Geltruda di Gesù Maria, cit.: 2r.

${ }^{86}$ Ibidem: $2 \mathrm{v}$.
} 
accreditando un modello di santità femminile intessuto di eroismo e soffernza. ${ }^{87}$ Per ben due volte scampata alle punture di uno sciame di api, la piccola Timotea riceve il dono meraviglioso di gustare il miele direttamente versato da esse nella sua bocca: «un prodigio che solo si racconta di Omero e di S. Ambrogio [...] Ma in Geltruda che non doveva insegnare né scrivere essendo donna, e senza studio di Lettere non poteva altra cosa significare se non che il Choro delle Vergini a Dio consacrate, secondo S. Ambrogio alle api rassomigliate quasi che mellificando cantino e cantando faccino il mele»..$^{8}$

Pia, obbediente, per nulla vanitosa, debole e fiacca per le infermità ma vigorosa quando «ragionava di Dio», la giovane Timotea, ben presto orfana di padre, si addentrava nella vita sotto l'occhio vigile del nonno paterno, una sorta di «patriarca» che decideva sia dei destini della nuora e della sua prole che di quelli dei propri figli e figlie: gli uni maritati con donne scelte con cura, le altre destinate per lo più al chiostro. Come quella zia, entrata nel monastero agostiniano di S. Scolastica che, come abbiamo visto nelle prime pagine, rimase talmente affascinata dalla lettura di una biografia di S. Teresa, da chiedere alla madre di destinare i propri averi alla fondazione di un monastero.

Colpisce in questa biografia, e soprattutto nella prima parte dedicata agli anni precedenti la monacazione di Timotea, l'attenzione portata dall'autore sul gruppo di donne che ruotano intorno ad essa, madre, zie e cugine, spiritualmente assistite dal gesuita Giacomo Cassia: «uomo dotto stimato da tutta Malta e dal vescovo Caglino di cui era confessore ordinario», a cui il capo famiglia si rivolge perché indaghi sulla vocazione delle fanciulle, incerte - come l'aristocratica Maria Maddalena di sant'Agostino - se prendere marito o divenire sponsa Christi. E ciò anche in preparazione dei capitoli matrimoniali di una di esse, Clara, che all'improvviso scelse di rinunciare alle nozze perché «non voleva esser causa d'un impedimento alla fondatione di un Monasterio et havere perciò a dar conto nel giorno del Giudizio che per lei restò indietro un'opera di servizio di Dio così grande»..$^{89}$

Non è certamente il caso di richiamare il dibattito svoltosi nella storiografia religiosa di genere italiana sull'opportunità offerta dal cattolicesimo alle donne che, a differenza delle protestanti, potevano trovare nel monastero il luogo in cui esprimere talenti, capacità e reti di relazioni, inconciliabili con

${ }^{87}$ Cfr. Cabibbo, S. Compare, C. 2012. La santità martiriale feminile tra modello in Guazzelli G., Michetti R., Scorza Barcellona F. (eds.), Cesare Baronio tra santità e scrittura storica: e paradigma, 223-248. Roma: Viella.

${ }^{88}$ Brieve relazione della Vita Morte e Miracoli della Madre Soro Geltruda di Gesù Maria, cit.: 3v.

${ }^{89}$ Brieve relazione della Vita Morte e Miracoli della Madre Soro Geltruda di Gesù Maria, cit.: 7v. 
la vita matrimoniale.$^{90}$ Ciò che è certo è che entrambe le agiografie esaminate propongono una tipologia di donna e di religiosa che, come la fondatrice spagnola, sceglie in piena coscienza il suo destino, pur avendo a disposizione altre alternative.

Fu questo il caso di Timotea e le sue congiunte: la madre e due zie. Quaranta giorni dopo la morte del «patriarca», risolute a fondare un monastero, entrarono nella casa di S. Giuseppe, una povera casupola adibita fino a poco tempo prima a rifugio per le malmaritate, ma allora disabitata e concessa dal vescovo alle quattro donne. Due anni dopo esse fecero «atto pubblico di donazione irrevocabile di quanto havevano e che fossero per havere al Monasterio edificando e costruendo» ${ }^{91} \mathrm{e}$, in attesa del breve di approvazione da Roma e non ancora professe, ascoltarono con grande profitto le prediche dei carmelitani scalzi sulla vita e le opere di Teresa d'Avila, stabilendo di professare la Regola della fondatrice spagnola sotto la giurisdizione dell'Ordine. Scrive l'agiografo:

«In publicarsi per Malta che le Signore ritirate nella casa di S. Gioseppe volevano essere Monache Teresiane sotto il governo delli Padri Scalzi del Carmine e fondare ivi novo convento, si mosse tutto l'inferno, sì che per questa causa patirono molte contradittioni e travagli delli quali il maggiore di tutti fu che essendosi il Prelato ingelosito della sua Jurisditione mutò parere né volse mai consentire à che si fondasse in Malta Monasterio di Monache esenti dall'ordinario sotto governo e Jurisdittone dei nostri Padri». ${ }^{92}$

La scelta diventava dunque quella di porsi sotto il governo dell'ordinario o di lasciare Malta e professare nel monastero teresiano di un'altra città; fu approvata questa seconda opzione con il parere favorevole del confessore e dei padri carmelitani, sicché dopo sette anni di permanenza nella casa di S. Giuseppe, Timotea di undici anni e le sue più adulte parenti si imbarcarono per Palermo nell'agosto del 1632, affrontando un viaggio pieno di «burrasche e crisi grandi di mare».

Piuttosto silente sino a questo momento, docile presenza al seguito delle decisioni della madre e delle zie, la fanciulla, sbarcata nella capitale del viceregno, mostra una volontà e un'intraprendenza fino ad allora insospettabili, opponendosi ad una visita della città proposta dalle parenti dichiarando «Noi siamo venute per la Religione e non per vedere Palermo», e soprattutto rifiutando di entrare nello stesso monastero teresiano scelto

\footnotetext{
${ }^{90} \mathrm{Si}$ veda a proposito Zarri, G., Scaraffia, L. 1994. Introduzione in Donne e fede. Santità e vita religiosa in Italia, cit., V-XVI.

${ }_{91}^{91}$ Brieve relazione della Vita Morte e Miracoli della Madre Soro Geltruda di Gesù Maria, cit., 8r.

${ }^{92}$ Ibidem: $8 \mathrm{v}$.
} 
dalla madre - quello dell'Assunzione - preferendo quello di S. Teresa che si apprestava ad accogliere le zie. «In questo mondo non ho altro bene che mia Madre - tramanda il biografo - se non lascio mia Madre per Dio, che cosa lasciarò io?». ${ }^{93}$

La vita nel monastero, le malattie, le sofferenze fisiche e spirituali, celebrate dai biografi delle tante serve di Dio morte in odore di santità, restano quasi in secondo piano in questa Vita che esprime la sua singolarità nella semplice fisionomia della protagonista - morta a quasi quarantré anni di cui più di ventitré passati in religione - che poco più che bambina si era lasciata alle spalle la sua isola e la lingua che vi si parlava, le persone e i luoghi dino ad allora frequentati, per approdare nell'aristocratico monastero di S. Teresa. I miracoli di guarigione di persone comuni, registrati dopo la sua morte, alludono forse alla diffusione dell'esperienza teresiana oltre i confini segnati delle aristocrazie palermitane, il cui modello di religiosità, intriso di pietà e fasto, aveva reso i loro monasteri e chiese «mausolei e custodi di genealogie di nobiltà e santità insieme». ${ }^{94}$

\section{BIBLIOGRAFÍA}

Alvarez, T., Fortes, A., Antolín, F., Giordano, S., Morgain, S.M. e Velasco, B. (eds.). 1995 Constituciones de las Carmelitas Descalzas (1562-1607). Roma: Teresianum.

Aringhi, P. 1885. «Vita inedita del Padre Francesco Soto» San Filippo Neri, gennaiofebbraio: 7-8; marzo-aprile: 4-6.

Bazzano, N. 2011. Moncada, Ugo, Dizionario Biografico degli Italiani. Vol. 75: 578-583, Roma: Istituto dell'Enciclopedia Italiana.

Bettini, G. e Franks, M. (eds.). 2014 . La chiesa di Santa Maria di Nazareth e la spiritualità dei carmelitani Scalzi a Venezia, 2014. Venezia: Marcianum Press.

Biagio della Purificazione. 1703. Vita dell'insigne Serva di Dio la Madre Maria Maddalena di S. Agostino, carmelitana Scalza nel Monastero di S. Anna e S. Teresa a Palermo, Roma: Stamperia e Gettaria del Zenobi.

Borromeo, A. 2003. «Filippo II e il Papato», in L. Lotti e R. Villari (eds.), Filippo II e il Mediterraneo: 477-535. Roma-Bari: Laterza.

Cabibbo, S. 1995. «Un'opera storica encomiastica nella sicilia del Seicento: I «ritratti della prosapia et heroi Moncadi» di Agostino della Lengueglia», in F. Benigno e C. Torrisi (eds.), Città e feudo nella Sicilia moderna: 137-152. Caltanissetta: Salvatore Sciascia editore.

\footnotetext{
${ }^{93}$ Ibidem: $10 \mathrm{v}$.

${ }^{94}$ Giarrizzo, Alla corte dei Moncada, cit. p. 436.
} 
Cabibbo, S. e Compare, C. 2012. «La santità martiriale femminile tra modello e paradigma», in G. Guazzelli, R. Michetti e F. Scorza Barcellona, Cesare Baronio tra santità e scrittura storica: 223-248. Roma: Viella.

Cabibbo, S. e Modica, M. 1989, La santa dei Tomasi. Storia di suor Maria Crocifissa (1645-1699), Torino: Einaudi.

Collectio scriptorum Ordinis Carmelitarum excalceatorum utriusque congregationis et sexus P. F. Bartholomei a S. Angelo Provinciae Longobardicae opera et solertia exarata, vol. I, Savona: Typographia Ricci.

Contreras J. 2009. «Procesos culturales hegemónicos: de religión y religiosidad en la España de Antiguo Régimen». Historia social, 35: 3-22.

Della Lengueglia A. 1652. Ritratti della prosapia et heroi Moncadi nella Sicilia. vol. II. Ritratto undecimo, Di donna Luisa Moncada, contessa di Santa Gadea e Buendia, poi monaca scalza carmelitana. Valenza: Vincenzo Sacco impressor viceregio.

Di Blasi, G.E. 1791. Storia cronologica dei viceré, luogotenenti e presidenti del Regno. Palermo: Stampe di Solli.

Di Matteo, S., 2002, Palermo, storia della città dalle origini ad oggi, Palermo: Kalos.

Dolfi, P.S. 1990. Cronologia delle famiglie nobili di Bologna. Bologna: Ed. Forni.

Fernández de Mendiola, D. 2011, El Carmelo teresiano en la historia. Una nueva forma de vida contemplativa y apostólica, Dos Congregaciones del Carmelo Descalzo. Desarrollo paralelo y visiones dispares (1597-1650). Vol. III. Roma: Instituto Histórico Teresiano.

Fini, M. 2007. Bologna sacra, Bologna: Pendragon.

Fondazione e traslazione del Monastero di Santa Teresa [...] scritte dalla Madre Maria Maddalena di S. Agostino [...] ed elogi di Nove Religiose morte sino a questo tempo in detto Monastero, cavati dagli scritti del Rev. P. F. Gio. Paulo dell'Epifania, Carm. S. cronista della provincia di Sicilia, Venezia: Turrini, 1672.

Fortes, A. (ed.). 1985. Monumenta Historica Carmeli Teresiani Subsidia 3, Acta Definitorii Generalis OCD Congregationis S. Eliae, Libro I, Regesta Actorum 16171627. Romae: Teresianum.

Fortes, A. OCD. 1988, 1989 y 1991. «Textos constitucionales de las Carmelitas Descalzas». Monte Carmelo 96 (1988): 509-550; 97 (1989): 88-125 e 483-518; 99 (1991): 335-363.

Ghezzi, C. 2006-2007. La Madonna delle Laste a Trento nel XVII secolo. Vita religiosa e aspetti artistici di una fondazione dei Carmelitani scalzi. Tesi di Laurea sotto la direzione della prof.ssa Elisabetta Marchetti, Università di Bologna Facoltà di Conservazione dei Beni culturali, A.A. 2006-2007.

Gianninoto, G. 1986. Mistero che attira. Per una storia del Carmelo teresiano in Sicilia. Villasmundo: Noviziato di Locomonico. 
Giarrizzo, G. 1999. «Alla corte dei Moncada». Annali di Storia moderna e contemporanea, 5: 429-436.

Giordano, S. 1991. Domenico di Gesù Maria Ruzola (1559-1630). Un carmelitano scalzo tra politica e riforma nella chiesa posttridentina, Roma: Teresianum.

Giordano, S. 1995, Costituzioni delle Carmelitane scalze d'Italia, 1593; in Costituciones de las Carmelitas Descalzas. Roma: Teresianum.

Janke-Leutzsch, G. 1992. «Clara Staiger, la priora», in G. Calvi (ed), Barocco al femminile: 97-126. Roma-Bari, Laterza.

Laudani, S. 2008. Lo Stato del Principe: I Moncada e i loro territori. Palermo: Sciascia.

Ligresti, D. 2010. «Centri di potere urbano e monarchia ispanica nella Sicilia del XV-XVIII secolo», in J. Martinez Millan y M. Rivero Rodríguez (eds.), Centros de poder italianos y monarquía hispanica (siglos XV-XVIII). vol. III: 287-329. Madrid: Ediciones Polifemo.

Macca, V. 2008. «Anna di S.Bartolomeo (Anna Garcia) beata, monaca OCD (15491626)», in Dizionario carmelitano, diretto da Boaga E. O.Carm - Borriello L OCD. Roma: Città Nuova.

Marchetti, E. 2000. «Il Carmelo scalzo e gli oratoriani a Roma» Archivio della Società di storia patria, 123: 105-131.

Marchetti, E. 2001, Le prime traduzioni italiane delle opere di Teresa di Gesù nel quadro dell'impegno papale post-tridentino, Bologna: Lo Scarabeo.

Marchetti, E. 2005. «La riforma del Carmelo scalzo tra Spagna e Italia». Dimensioni e problemi della ricerca storica, $\mathrm{n} .1$, Identità religiose e identità nazionali in età moderna, M. Caffiero, F. Motta, S. Pavone (eds): 13-24.

Marchetti, E. 2013. «L'Ordine dei Carmelitani scalzi e i Santuari tra XVI e XVIII secolo», in L.M.Olivieri (ed), Ordini religiosi e santuari in età moderna e contemporanea: 207-221. Bari: Edipuglia.

Marchetti, E. 2014. «Venezia e Santa Maria di Nazareth: tappe significative nello sviluppo culturale e nella diffusione della realtà scalza», in G. Bettini e M. Franks, (eds.). La chiesa di Santa Maria di Nazareth e la spiritualità dei carmelitani Scalzi a Venezia: 101-112. Venezia: Marcianum Press.

Mazzarese Fardella, E. 2007. «Aspetti della religiosità in Sicilia nei secoli XVI-XVII. I Fardella-Paceco». Archivio Storico Siciliano, serie IV, XXXIII, 2007: 7-42.

Medioli, F. 1997. «La clausura delle monache nell'amministrazione della Congregazione romana sopra i Regolari», in Il monachesimo femminile in Italia dall'altro medioevo al secolo XVII: 249-282. Verona: Il segno dei Gabrielli.

Mejia Maya, R. 1995. Las fundaciones de las Camelitas Descalzas en Italia, Malta y paises balcanicos (1590-1992). Carmelos en el mundo. Burgos: Editorial Monte Carmelo. 
Monumenta Historica Carmeli Teresiani (MHCT), III, doc. 349: 314-315.

Monumenta Historica Carmeli Teresiani 11, Acta Capituli Generalis OCD Congregationis S. Eliae, vol. I, Antonio Forte (ed.) 1990. Romae: Teresianum.

Morgain, S.M. 1995. Pierre de Bérulle et les Carmélites en France, La querelle du gouvernament, 1583-1629. Paris: Cerf.

Moriones, I. 1968. Ana de Jesús y la herencia teresiana, ¿Humanismo cristiano o rigor primitivo? Roma: Teresianum.

Motta, F.- Pavone, S., 2005. «Per una storia comparativa degli ordini religiosi». Dimensioni e problemi della ricerca storica, n. 1, Identità religiose e identità nazionali in età moderna, M. Caffiero, F. Motta, S. Pavone (eds): 13-24.

Nocella, P. L. 2010. «Lealtad a la corona y ascenso social de una familla siciliana», in G. Levi e R. Rodríguez Pérez (eds.), Familias, jerarquización y movilidad social: 341-352., Murcia: Universidad de Murcia.

Novi Chavarria, E. 2001. Monache e gentildonne, un labile confine. Poteri politici e identità religiose nei monasteri napoletani (secoli XVI-XVII). Milano: Franco Angeli.

Pinzarrone, L. 2014. «La politica delle fondazioni feudali nella Sicilia del XVII secolo: procedure, controversie, giurisdizioni», Storia urbana, 142: 5-20.

Raines, D. 2014. «La lobby cittadina dei Carmelitani Scalzi nella Venezia settecentesca», in G. Bettini e M. Franks, (eds.). La chiesa di Santa Maria di Nazareth e la spiritualità dei carmelitani Scalzi a Venezia: 77-199. Venezia: Marcianum Press.

Rapley, E. 1990. The 'Dévotes'. Women and Church in Seventeenth Century France, MC Gill-Queen's University Press Montreal \& Kingston.

Regola e Costitutioni delle religiose primitive dell'Ordine della Gloriosa Vergine Maria del monte Carmelo, In Genoua, per gli Heredi di Gir. Bartoli, MDXCIII.

Riforma de' Scalzi di N.S. del Carmine dell'osservanza primitiva fatta da S. Teresa di Giesù nell'antichissima Religione fondata dal Gran Profeta Elia, scritta e stampata in castigliano dal M.R.P.F. Giuseppe di Santa Teresa suo historico generale e tradutta nell'italiana da un Religioso del medesimo Ordine, 1701. Parma: per Alberto Pazzoni e Paolo Monti, tomo quarto.

Roggero, A. 1984. Genova e gli inizi della Riforma Teresiana in Italia (1584-1597), Roma: Teresianum.

Roggero, A. 1996. Origini della presenza carmelitana maschile e femminile a Genova, in S. Giordano - C. Paolocci (eds), Nicolò Doria. Itinerari economici, culturali e religiosi nei secoli XVI-XVII tra Spagna, Genova e l'Europa, II: 315-331. Roma: Teresianum.

Ruzza di, O. 1987. Sintesi storico-cronologica della Provincia romana dei Padri Carmelitani Scalzi. Roma: Edizioni OCD. 
Sanfilippo M., 1992. «Doria Giannettino», en Dizionario Biografico degli Italiani, vol. 41: 345-348. Roma: Istituto dell'Enciclopedia Italiana.

Santa Maria, Francesco di. 1654-1662. Riforma degli Scalzi di Nostra Signora del Carmine dell'Osservanza primitiva....vol. I trad. di Gasparo di San Michele, Genova 1654; vol. II trad. Girolamo di Santa Teresa, Bologna 1662.

Scalisi L. 2004. Il controllo del sacro. Poteri e istituzioni concorrenti nella Palermo del Cinque e Seicento, Roma: Viella.

Scaraffia L., Zarri G. (ed.). 1994. Donne e fede: 177-225. Roma-Bari: Laterza.

Sérouet, P. (ed.). 1971. Quintanadueñas. Lettres de Jean de Brétigny (1556-1634). Louvain: Revue d'Histoire Ecclésiastique.

Sérouet, P. 1974. «Jean de Brétigny (1556-1634). Aux origine du Carmel de France, de Belgique et du Congo» Bibliothèque de la Revue d'Histoire Ecclésiastique, 60.

Serouet, P. 1974, Jean de Brétigny, aux origines du Carmel de France, de Belgique et du Congo, Louvain.

Sierra, T. 1996. Nicolás Doria, innovador del gobierno de la Reforma carmelitana, in S. Giordano e C. Paolocci (eds), Nicolò Doria. Itinerari economici, culturali e religiosi nei secoli XVI-XVII tra Spagna, Genova e l'Europa, II: 219-235. Roma: Teresianum.

Silverio di S. Teresa OCD, «Constituciones primitivas», in Biblioteca Mistica Carmelitana (BCM) 6: VII-XXX.

Spagnoletti, A. 1996. Principi italiani e Spagna nell'età barocca, Milano: Bruno Mondadori.

Sturm, S. 2002. L'eremo di Montevirginio e la tipologia del Santo Deserto, Roma: Gangemi Editore.

Weber,A. 1993. Teresa d'Avila e la retorica della femminilità, Firenze: Le Lettere.

Zarri, G. 2000. Recinti. Donne, clausura e matrimonio nella prima età moderna Bologna: Il Mulino.

Zarri, G. 1994. «Dalla profezia alla disciplina (1450-1650)», in Scaraffia L, Zarri G (ed.), Donne e fede: 177-225. Roma-Bari: Laterza. 